\title{
Performance Analysis of Non-Regenerative Massive-MIMO-NOMA Relay Systems for 5G
}

\author{
Di Zhang, Student Member, IEEE, Yuanwei Liu, Member, IEEE, Zhiguo Ding Senior Member, IEEE, Zhenyu \\ Zhou, Member, IEEE, Arumugam Nallanathan, Fellow, IEEE, and Takuro Sato Fellow, IEEE.
}

\begin{abstract}
The non-regenerative massive multi-input-multioutput (MIMO) non-orthogonal multiple access (NOMA) relay systems are introduced by this study. The NOMA is invoked with superposition coding technique at the transmitter and successive interference cancellation (SIC) technique at the receiver. In addition, a maximum mean square error (MMSE)SIC receiver design is adopted. With the aid of deterministic equivalent and matrix analysis tools, closed-form expression of the signal to interference plus noise ratio (SINR) is derived. To characteristic the performance of the considered systems, closed-form expressions of the capacity and sum rate are further obtained based on the derived SINR expression. Insights from the derived analytical results demonstrate that the ratio between the transmitter antenna number and the relay number is a dominate factor of the system performance. Afterwards, the correctness of the derived expressions are verified by the Monte Carlo simulations with numerical results. Simulation results also illustrate that: i) the transmitter antenna, averaged power value and user number display the positive correlations on the capacity and sum rate performances, whereas the relay number displays a negative correlation on the performance; ii) the combined massive-MIMO-NOMA scheme is capable of achieving higher capacity performance compared to the conventional MIMONOMA, relay assisted NOMA and massive-MIMO orthogonal multiple access (OMA) scheme.
\end{abstract}

Index Terms-Non-regenerative relay assisted systems, 5G, massive MIMO, NOMA, MMSE-SIC.

\section{INTRODUCTION}

$\mathbf{F}$ IFTH generation $(5 \mathrm{G})$ is brought up to tumble down the explosive increasing number of devices accessing to the internet [1]-[3]. Spectrum efficiency (SE), energy efficiency

Manuscript received March 12, 2017; revised June 28, 2017; accepted August 06, 2017.

Corresponding author: Z. Zhou (zhenyu_zhou@ncepu.edu.cn).

This work was partially supported by the National Science Foundation of China under Grant 61601181, the Fundamental Research Funds for the Central Universities under Grant 2017MS13, by the Beijing Natural Science Foundation under Grant 4174104, and Beijing Outstanding Young Talent under Grant 2016000020124G081.

The work of Z. Ding was supported by the UK EPSRC under grant number EP/L025272/1 and by H2020-MSCA-RISE-2015 under grant number 690750 .

D. Zhang and Z. Zhou are with the State Key Laboratory of Alternate Electrical Power System with Renewable Energy Sources, School of Electrical and Electronic Engineering, North China Electric Power University, Beijing, 102206, China (email: di_zhang@fuji.waseda.jp, zhenyu_zhou@ncepu.edu.cn). D. Zhang is also with the GITS/GITI, Waseda University, Tokyo, 169-0072, JP.

Y. Liu and A. Nallanathan are with the Department of Informatics, King's College London, Strand, London WC2R-2LS, UK (email: \{yuanwei.liu, arumugam.nallanathan\}@kcl.ac.uk).

Z. Ding is with the School of Computing and Communications, Lancaster University, LA1-4WA, UK (email: z.ding@lancaster.ac.uk).

T. Sato is with the GITS/GITI, Waseda University, Tokyo, 169-0072, JP (email: t-sato@waseda.jp).
(EE) and low latency processing are main focused topics for 5G's deployment and implementation [2], [4], [5]. Besides the prior key technologies with massive multi-input-multi-output (MIMO) [6], [7], small cell [2] and heterogeneous networks (HetNets) [5], non-orthogonal multiple access (NOMA) has received various attentions both in academia and industry for its SE performance merits [8]-[10]. The is due to the fact that NOMA can share the same frequency resource block (called as the sub-band [11] in the following content) by multiple users [11]. In literature, NOMA technologies are categorized by different domains, for instance, code domain NOMA ( e.g. sparse code multiple access (SCMA) [12], pattern division multiple access (PDMA) [8], [13]) and power domain NOMA [14]$[16]^{1}$. The idea of NOMA is to exploit the different quality of service (QoS) requirements of different users [17], [18]. At the receiver side, to decode the non-orthogonal transmit information, successive interference cancellation (SIC) was proposed [15], [19]-[22]. With SIC, users within good channel condition can eliminate other users' encoding information with higher orders of the same sub-band according to the allocated power value [19], [20], or with a reversed decoding order according to the signal to noise ratio (SNR) [15], [21] (or signal to interference plus noise ratio (SINR) [8]). Here in this study, the first scheme is adopted.

In the initial studies of NOMA, BS with single transmit antenna paradigm is investigated a lot. For instance, the two user scenario was propounded to compare with the orthogonal frequency division multiple access (OFDMA) [14]; it was proved that with equal power allocation, NOMA can achieve about $48 \%$ corresponding gain compared to OFDMA. The multiple user scenario with randomly deployed location problem was raised in [15], in which the system outage performance was discussed. Additionally, randomly deployed user problem was addressed with a large scale underlay cognitive radio network architecture by [23], the closed-form expression of the outage probability was derived. NOMA was combined with simultaneous wireless information and power transfer (SWIPT) in [24]; it was proposed that the users nearby the BS performing as the energy harvesting relays to assist cell edge users' transmission.

On the capacity study of multiple user NOMA scenario (also known as MIMO-NOMA), the capacity performance was compared between the MIMO-NOMA and conventional MIMO orthogonal multiple access (MIMO-OMA) systems

\footnotetext{
${ }^{1}$ Note that here in this paper, while talking about NOMA, we are referring the power domain NOMA in the following content.
} 
in [25]. Observation from this study witnessed that MIMONOMA was capable of achieving higher ergodic sum capacity performance compared to MIMO-OMA. The dynamic power allocation mechanism was proposed in [26] to maximum the overall cell capacity. The MIMO-NOMA was compared with MIMO-OMA in [27], numerical results found that MIMONOMA surpassed MIMO-OMA for two user and multiple user scenarios. The power allocation with proportional fairness scheduling (PFS) scheme was introduced for the downlink transmission of two user scenario in [28]; it was found that PFS can maximize the minimum normalized rate with proportional fairness and small variation of the transmission rates.

Latest, massive-MIMO-NOMA is noticed to further boost up 5G's capacity and sum rate performances, although fewer studies have been done in this regard. Prior studies on massiveMIMO-NOMA can be found as: The frequency multiplexing method (also called as NOMA orthogonal frequency division multiplexing (NOMA-OFDM)) was proposed firstly by [11]. Compared to prior studies, this method can further enhance the system's sum rate performance. Moreover, a limited feedback scheme for massive-MIMO-NOMA was proposed by [29]; it was proved that such a system can be decomposed into multiple single-input-single-output (SISO) channels. The efficient antenna selection and user scheduling algorithms were investigated in [30] to maximize the sum rate; numerical results demonstrated that the proposed joint antenna and user (AU) algorithm achieved the same performance of existing methods with a lower complexity.

As discussed, massive-MIMO-NOMA can further enhance the system capacity and sum rate performances in $5 \mathrm{G}$. Yet it is noticed that with the inevitable denser cellular deployment of massive MIMO [31], alternative architecture design to progressively enlarge the coverage area is of crucial significance. In this regard, the relay system model attracts more and more attentions [32]; where the relay can be either regenerative or non-regenerative [33], [34]. Generally, the non-regenerative relay system is simple and of better latency performance [33]. In prior studies, the MIMO minimum mean square error (MMSE) non-regenerative systems were investigated based on the local channel state information (CSI) by [34]. One optimal power allocation mechanism was proposed in [35]. The study in [36] introduced a stochastic robust collaborative beamforming method. On the other hand, NOMA with relay systems also received significant attentions recently. For instance, the relay assisted NOMA systems' resource allocation problem was investigated in the study of [37], where the proposed matching algorithm can provide sub-optimal solution within an affordable time. The adaptive transmission with buffer assisted NOMA relaying system was proposed in [38], where with the introduced optimal mode selection mechanism, the system throughput was improved.

\section{A. Motivation and Contributions}

While the aforementioned studies laying solid foundation on both MIMO-NOMA and relay assisted NOMA systems, the research contributions in terms of massive-MIMO-NOMA, and method to enlarge its coverage area, are still in their fancy. This inspires us to develop this treatise. Other than the prior studies, in this paper, the massive-MIMO is adopted in the relay assisted NOMA system to enhance the system capacity performance ${ }^{2}$. Additionally, the NOMA-OFDM [11] is used as well to further increase the sum rate performance. To highlight the merits of this proposal, the capacity and sum rate performances of massive-MIMO-NOMA with prior massive-MIMO-OMA are compared within the same relay assisted non-regenerative systems. The capacity and sum rate performances of this study are compared with prior MIMONOMA and relay assisted NOMA systems as well. Additionally, other than the mentioned studies with optimal power allocation, beamforming design and user clustering analysis, here to settle down the more complex analysis, deterministic equivalent and matrix analysis tools are invoked to get the closed-form capacity and sum rate expressions.

The contributions of this paper can be summarized as follows:

- We propose a novel non-regenerative massive-MIMONOMA relay system model. Moreover, the MMSESIC decoding scheme and multiple sub-band frequency regime are elaborated as well. Compared with prior MIMO-NOMA [25], [27] or relay assisted NOMA studies [37], [38], the capacity and sum rate performances are further enhanced.

- We derive an exact closed-form SINR expression. Afterwards, we achieve the closed-form expressions of system capacity and sum rate. The analytical results clearly demonstrate that other than the findings with prior studies (transmit antenna number, allocated power, channel noise etc.), the ratio between the number of antenna at transmitter and the number of relay is another dominant factor of the system performance.

- We demonstrate that the impact of transmit antenna number and user number's positive correlation effects on system performance become weaker while the number of antenna grows large. Yet by increasing SNR, higher performance can always be obtained. In addition, compared to relay assisted massive-MIMO-OMA, the proposed relay assisted massive-MIMO-NOMA scheme is capable of achieving about 30\% 50\% capacity increment in each sub-band by each user. This capacity increment merit becomes even obvious while the transmitter antenna number growing large.

\section{B. Organization and Notations}

The remaining structure of this paper is organized as follows: Section II is the non-regenerative massive-MIMONOMA relay assisted system model description. The SINR, capacity and sum rate are investigated with closed-form expressions by section III. Numerical results are provided to verify the deduction accuracy and the different variables' (transmit antenna number, user number, relay number, allocated power

\footnotetext{
${ }^{2}$ Note that in this work, the system capacity means the sum capacity of the system, not each channel capacity.
} 
values) influences to the system performance by section IV. Section V finally concludes the study.

All through this paper, the uppercase boldface letters denote the matrices; lowercase boldface letters are the vectors; and normal letters are the scalar quantities, respectively. Furthermore, $\mathbb{C}$ is the complex set; $\mathbb{R}$ yields the real set; $\mathbf{A}^{H}$ denotes the Hermitian transposition of a matrix $\mathbf{A} ; \mathbf{A}_{i, j}$ is the $(i, j)$ entry of a matrix $\mathbf{A}$ with $i$ th raw and $j$ th column; $\operatorname{tr}(\mathbf{A})$ stands for the trace of $\mathbf{A} ; \mathbb{E}(\mathbf{A})$ is the expectation of the matrix $\mathbf{A}$; and $\mathbf{A}^{-1}$ is the inverse transpose of matrix $\mathbf{A}$, respectively. In addition, $\stackrel{a . s .}{\longrightarrow}$ means almost surely approaching; and $\circ$ is the Hadamard product. Finally, sup and inf respectively denote the supremum and infimum.

\section{The System Model}

In this section, the system model and received information are addressed. The non-regenerative relay system model for massive-MIMO-NOMA transmission is introduced first with a multiple sub-band scheme. After that, the user at receiver side can invoke the MMSE-SIC decoding method to retrieve its transmitted information.

\section{A. System Model Description with MMSE-SIC Decoding De- sign}

Detail information of the non-regenerative massive-MIMONOMA relay systems will be elaborated first. As known, typically in massive-MIMO systems, there are large number of transmit antennas that serving fewer users [6], [39], [40]. With this knowledge in hand, the non-regenerative massive-MIMONOMA relay systems proposed in this study are described by Fig. 1, with both reality and theoretically simplified descriptions. It is supposed that there are $M$ transmit antennas and $K$ users with $R$ relays of the non-regenerative massiveMIMO-NOMA relay systems, as depicted in this figure. The transmission procedure can be taken as two phases. In the first phase, the target information is encoded with NOMA encoding method and handed over to the relays from the transmitter. Afterwards, in the second phase, the encoded information will be delivered to the terminal side by the relays, where the AF relay model is assumed in this study for the sake of simplicity. The merit of this system lies in the fact that it can provide even faster transmit rate to the cellular edge users with the help of relay. Moreover, the relays can either be the assigned access point (AP) or users within the cellular inner area, as shown by Fig. 1(a). With the AF mode, signal re-generation is not necessary, which is a simple and efficient model to enlarge the coverage area and improve the QoS performance for cellular edge users.

In this relay assisted non-regenerative massive-MIMONOMA system, within each sub-band, different power values are allocated for different users with NOMA encoding scheme. It is further assumed that user orders are given in line with an increasing allocated power value order of each user within each sub-band. The NOMA concept with orthogonal frequency division multiplexing (OFDM) [11] while employing multiple sub-bands in this study is indicated by Fig. 2. This is because that in $5 \mathrm{G}$, even wider bandwidth can be assigned to meet

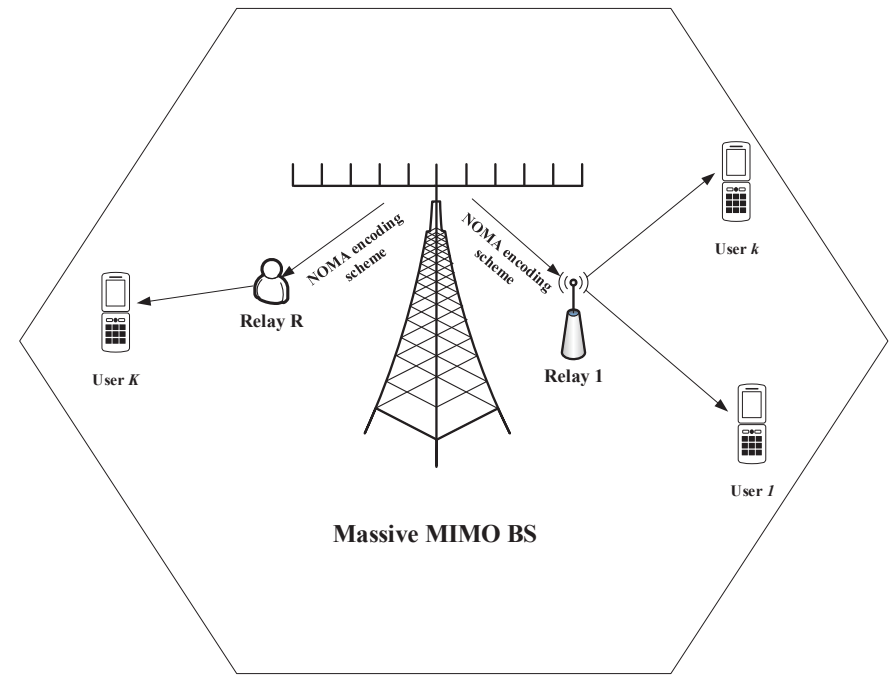

(a) The reality non-regenerative relay assisted massive-MIMO-NOMA system.

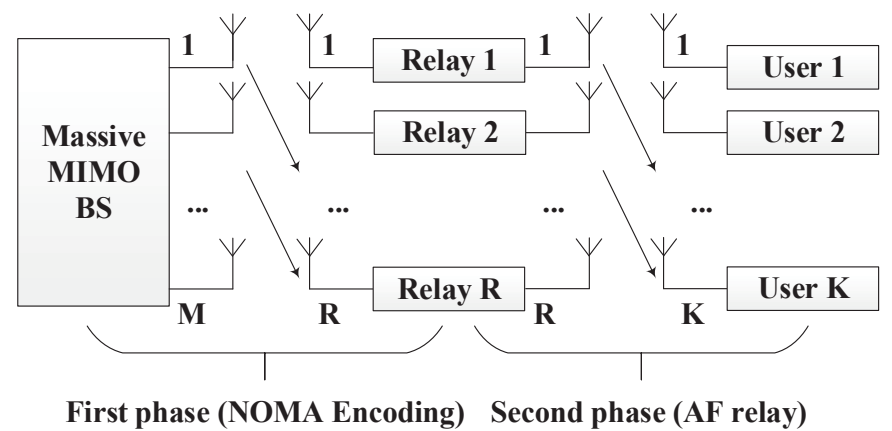

(b) The theoretically simplified system model.

Fig. 1. Demonstration of the non-regenerative relay system design ( $M$ transmitted antenna, $R$ relay, $K$ user).

the even fast transmission rate requirement [41], [42]. In this case, it will be a waste of resource method to allocate the entire bandwidth for one link from transmitter to receiver side, especially with much lower transmission rate requirement. Indeed, this problem can be partly alleviated via NOMA. However, by employing NOMA and OFDM at the same time, the frequency resources can be more effectively used. In addition, NOMA-OFDM can be a flexible scheme, in which the users can employ multiple sub-bands for their transmission if needed. That is, it can allocate more sub-bands to the users with fast transmission rate requirements. On the contrary, less ones for the users with lower transmission rate requirements.

As shown by Fig. 2, the length of each rectangle is used to denote one sub-band. It is assumed that the bandwidth is divided into $S$ sub-bands. In addition, height of each rectangle yields the power value allocated to each user $k$ (where $1 \leq k \leq K$ ) with NOMA encoding scheme [14] $]^{3}$. Without loss of generality, we further assume that within each subband, $K$ users exploiting the increasing power values for their

\footnotetext{
${ }^{3}$ Note that for the sake of compactness, we follow the prior studies in [11]. In addition, equal power value and order are assumed for each user $k$ amongst multiple sub-bands. In contrast, in each sub-band, different users are allocated with different power values. Additionally, the user orders are the same amongst different sub-bands. This is called as the same-position-same-power NOMA power allocation scheme.
} 
NOMA-OFDM scheme:

Superposition and power allocation within multiple sub-bands

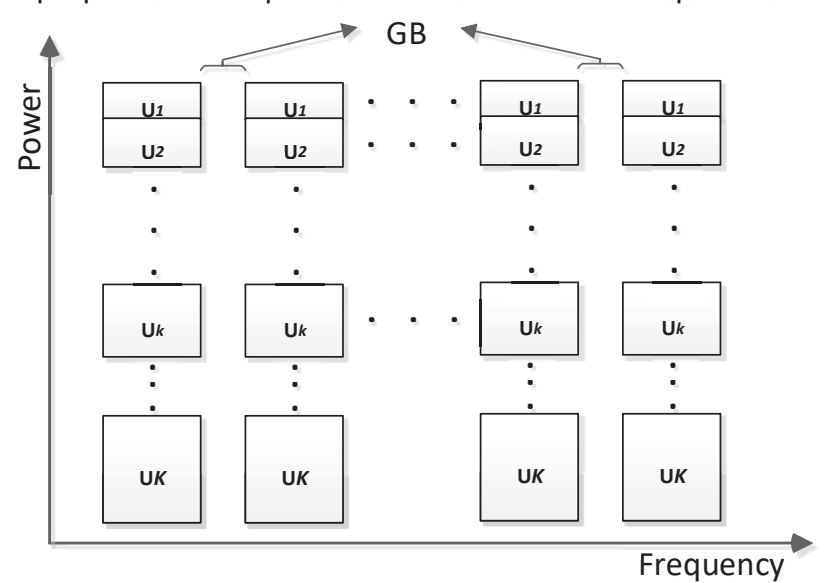

Fig. 2. Demonstration of the NOMA-OFDM scheme.

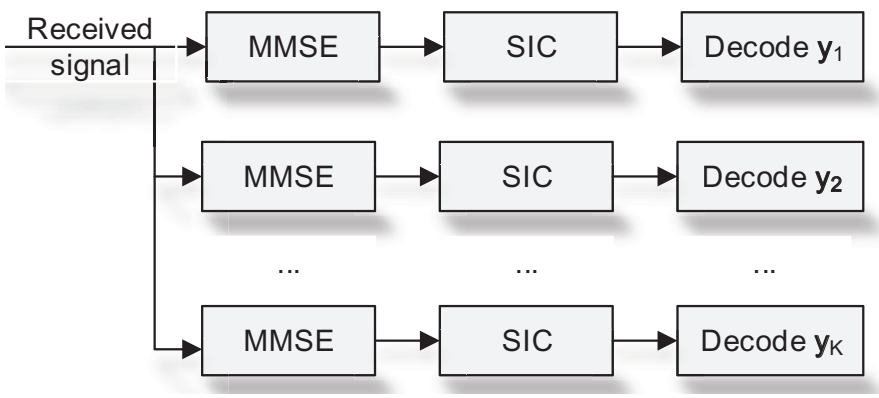

Fig. 3. Demonstration of the MMSE-SIC decoding method within one time interval.

transmission while user order growing. This is indicated by Fig. 2 with the increasing heights of different rectangles from user 1 to user $K$ of each sub-band. Furthermore, to alleviate the interferences from neighboring sub-bands, the guard band (GB) method [43] is adopted, as shown by Fig. 2. Generally, in wireless as well as wired communications, the GB is a part of unused spectrum between different spectrum bands [44], [45]. The benefit of GB lies in the fact that it can isolate the inter-channel interferences [45]. This is especially useful in the complex communication systems that the orthogonal correlation between channels can not be exactly achieved.

While decoding the received information at the received side, the MMSE-SIC decoding method is introduced. Here to elaborate this mechanism, one example is given by Fig. 3. With MMSE and GB amongst different sub-bands, it is assumed that the inter-channel interferences are perfectly eliminated. In addition, SIC removes the co-channel interferences from other users with higher power values, and keeps the co-channel interferences with lower power values as interferences for each user $k$. This will be further clarified by the following analysis.

\section{B. Signal Model}

In this study, the channel matrix from transmitter to receiver is assumed to be $\mathbf{H}$ (specific structure of $\mathbf{H}$ will be given by the following analysis). The received signal then can be given as

$$
\mathbf{y}=\mathbf{H x}+\mathbf{n},
$$

where $\mathbf{x}$ is the transmitted information via NOMA encoding method, and $\mathbf{n}$ the channel noise.

At the first phase of this non-regenerative relay system, it is assumed that $\mathbf{H}_{1} \in \mathbb{C}^{M \times R}$ is the channel matrix. In this study, to investigate the capacity and sum rate performances, the channel condition (channel matrix eigenvalue) is assigned to $K$ user with an increasing order. That is, the $K$ th user (where each user is denoted as $k$ with $1 \leq k \leq K$ ) is assigned the best channel condition while the 1st user the worst channel condition. Furthermore, as discussed aforementioned, we assume that multiple sub-bands with a total sub-band number $S$ are used. In this case, without loss of generality, by adopting the matrix expression, received signal after the first phase within each sub-band can be given as

$$
\mathbf{y}_{1}=\mathbf{H}_{1}^{H} \mathbf{x}+\mathbf{n}_{1},
$$

where $\mathbf{x}$ denotes the transmit signal with $\mathbf{x}=\sum_{i=1}^{S} \sqrt{\mathbf{P}}_{i} \circ \mathbf{s}$. Furthermore, $\mathbf{P} \in \mathbb{C}^{M \times S}$ is denoted as the allocated power matrix for all NOMA users from $M$ massive-MIMO transmit antennas of a total sub-band number $S$. In addition, $\mathbf{s}$ is the transmitted signal vector with $\mathbb{E}\left\{\mathbf{s s}^{H}\right\}=\sigma_{s}^{2} \mathbf{I}_{M} ; \mathbf{n}_{1}$ is the noise vector with $\mathbb{E}\left\{\mathbf{n}_{1} \mathbf{n}_{1}^{H}\right\}=\sigma_{1}^{2} \mathbf{I}_{R}$. This will bring in the received information in all sub-band rephrased as

$$
\mathbf{y}_{1}=\sum_{i=1}^{S} \mathbf{H}_{1}^{H} \mathbf{x}_{i}+\mathbf{n}_{1},
$$

where $\mathbf{x}_{i}=\sqrt{\mathbf{P}}_{i} \circ \mathbf{s}$.

Additionally, by following similar analytical procedure of the first phase system, received signal at the receiver side after the two phases of this non-regenerative massive-MIMONOMA relay systems, will be

$$
\begin{aligned}
\mathbf{y}_{2} & =\mathbf{H}_{2}^{H} \mathbf{G}_{1} \mathbf{y}_{1}+\mathbf{n}_{2} \\
& =\underbrace{\mathbf{H}_{2}^{H} \mathbf{G}_{1} \mathbf{H}_{1}^{H}}_{\mathbf{H}} \mathbf{x}+\underbrace{\mathbf{H}_{2}^{H} \mathbf{G}_{1} \mathbf{n}_{1}+\mathbf{n}_{2}}_{\mathbf{n}} \\
& =\mathbf{H} \mathbf{x}+\mathbf{n} .
\end{aligned}
$$

where $\mathbf{H}_{2} \in \mathbb{C}^{R \times K}, \mathbf{G}_{1} \in \mathbb{C}^{R \times R}$, and $\mathbf{n}_{2}$ (with $\mathbb{E}\left\{\mathbf{n}_{2} \mathbf{n}_{2}^{H}\right\}=$ $\sigma_{2}^{2} \mathbf{I}_{K}$ ) yield the second phase channel matrix from the relay to the receiver, the linear relay pre-coder of the relay system [33], [46], and the noise vector of the second phase, respectively. As shown by this equality, for the sake of compactness, we can employ $\mathbf{H}$ as the "channel matrix" to denote the $\mathbf{H}_{2}^{H} \mathbf{G}_{1} \mathbf{H}_{1}^{H}$ of the non-regenerative massive-MIMO-NOMA relay systems. Additionally, $\mathbf{n}$ is invoked to denote the $\left(\mathbf{H}_{2}^{H} \mathbf{G}_{1} \mathbf{n}_{1}+\mathbf{n}_{2}\right)$, which is treated as the "channel noise" in this treatise. In the case, the signal transmission of the non-regenerate massiveMIMO-NOMA relay systems can be described with more general signal model. The analysis procedures and conclusions of multi-variable analysis and deterministic equivalent will be adopted in the performance analysis of the following section. 
While employing the massive-MIMO antenna for transmission, the received signal of each user $k$ at the receiver side, is described as

$$
\begin{aligned}
\mathbf{y}_{k} & =S \sqrt{p_{k}} \mathbf{H}_{2}^{H} \mathbf{G}_{1} \mathbf{H}_{1}^{H} \mathbf{s}+\mathbf{H}_{2}^{H} \mathbf{n}_{1}+\mathbf{n}_{2} . \\
& =S \sqrt{p_{k}} \mathbf{H}_{2}^{H} \mathbf{H}_{1}^{H} \mathbf{s}+\mathbf{H}_{2}^{H} \mathbf{n}_{1}+\mathbf{n}_{2} .
\end{aligned}
$$

Here $p_{i}, p_{k}$ denotes the power value within sub-band $i$ and arbitrary sub-band $k$. In addition, as shown by the last step of (5), the relay pre-coder matrix $\mathbf{G}_{1}$ is assumed to be an unit matrix. This is due to the fact that normally, the optimal solution of $\mathbf{G}_{1}$ is a linear pre-coder in the non-regenerative relay system [33]. Actually, the pre-coder design is another hot topic in non-regenerative MIMO relay systems, such as the study in [46]. However, here in this study, our focus is the massive-MIMO-NOMA capacity and sum rate performances. The optimal pre-coder design for this non-regenerative relay system is left for further study.

With the discussion above, the MMSE matrix that employed here can be given as [6], [47]

$$
\text { MMSE }=\sqrt{P_{k}} \mathbf{h}_{k}^{H} \mathbf{w}_{k}^{H}\left(\mathbf{W H P H} \mathbf{H}^{H} \mathbf{W}^{H}+\mathbf{R}_{n n}\right)^{-1},
$$

where $\mathbf{h}_{k}, \mathbf{W}_{k}$ denote the channel and pre-coder, respectively. After the MMSE-SIC, received information of each user $k$ can be given as

$$
\operatorname{MMSEy}_{k} / S=\frac{\sqrt{P_{k}} \mathbf{h}_{k}^{H} \mathbf{w}_{k}^{H}\left(\mathbf{W H P H} \mathbf{H}^{H} \mathbf{W}^{H}+\mathbf{R}_{n n}\right)^{-1} \mathbf{y}_{k}}{S},
$$

where $\mathbf{R}_{n n}$ is

$$
\mathbf{R}_{n n}=\sigma_{1}^{2} \mathbf{H}_{2} \mathbf{H}_{2}^{H}+\sigma_{2}^{2} \mathbf{I}_{K} .
$$

Afterwards, SIC is adopted for each user $k$ to cancel the user interferences with higher user orders while treating the other transmitted signals with lower user orders as the interferences. That is, while decoding at the receiver side with SIC, the $K$ th user's information is first retrieved while treating other user information as interference, then $(K-1)$ th user's information retrieved. The decoding is executed by repeating this procedure until the 1st user's information is decoded that without any interference from other user. Based on this, the capacity and sum rate analysis of this non-regenerative massive-MIMONOMA relay systems will be addressed by the following section.

\section{The CAPACITy And Sum Rate AnAlysis}

The system sum rate performance will be addressed in this section. To achieve the closed-form expression, analysis procedure in this paper is that: firstly, the random matrix theory with its analysis procedures and conclusions are applied here as the analytical tools; afterwards, SIC is addressed to obtain the final expression of the capacity; finally, closed-form expression of the sum rate is achieved by summing all user's capacity and multiplying it with the sub-band bandwidth value and its number $S$.

Beforehand, it is noticed from prior study in [47] that for the rectangular matrix $\mathbf{A}$ with entries independent and identically distributed (i.i.d.), the following assumptions holding: i)
$\mathbb{E}\left(\mathbf{A}_{i, j}\right)=0$ and $\mathbb{E}\left(\mathbf{A}_{i, j}^{2}\right)=1$; ii) the nonzero eigenvalues of $\mathbf{A} \mathbf{A}^{H}$ and $\mathbf{A}^{H} \mathbf{A}$ are the same.

Lemma 1: SINR expression: With the elaborated power allocation scheme of multiple sub-bands, at the receiver side, after MMSE, SINR expression of each user $k^{4}$ in the nonregenerative massive-MIMO-NOMA relay systems that denoting by $\gamma_{k}$ can be given as

$$
\gamma_{k}=\frac{p_{k}\left|\mathbf{h}_{k}\right|^{2} \mathbf{w}_{k}^{H}\left(\sum_{i=1}^{K} p_{i}\left|\mathbf{h}_{k}\right|^{2} \mathbf{w}_{i} \mathbf{w}_{i}^{H}+\mathbf{R}_{n n}\right)^{-1} \mathbf{w}_{k}}{1-p_{k}\left|\mathbf{h}_{k}\right|^{2} \mathbf{w}_{k}^{H}\left(\sum_{i=1}^{K} p_{i}\left|\mathbf{h}_{i}\right|^{2} \mathbf{w}_{i} \mathbf{w}_{i}^{H}+\mathbf{R}_{n n}\right)^{-1} \mathbf{w}_{k}} .
$$

Proof: Please see Appendix A.

With this expression in hand, the power allocation scheme with each sub-band for multiple users that discussed aforementioned in section II is further employed. By following the Shannon theory, achievable capacity of user $k$ is written as

$$
C_{k}=\log _{2}\left(1+\gamma_{k}\right)
$$

In this case, capacity of the non-regenerative massive-MIMONOMA relay systems can be given as

$$
C_{\text {sum }}=\sum_{k=1}^{K} \log _{2}\left(1+\gamma_{k}\right)
$$

Finally, the sum rate of the non-regenerative massive-MIMONOMA relay systems will be

$$
R_{\text {sum }}=S B \sum_{k=1}^{K} \log _{2}\left(1+\gamma_{k}\right)
$$

where $B$ yields the sub-band bandwidth value. As clearly shown here, the main task is to tackle down $\gamma_{k}$, whose closedform expression will be given by two cases in the following analysis.

\section{A. The SINR Analysis}

To derive the closed-form expression of the $\gamma_{k}$, we will introduce two lemmas that derived from prior studies, which are given as follows.

Lemma 2: On condition that $\mathbf{A} \in \mathbb{C}^{N \times N}$, and $\mathbf{x} \sim$ $\mathcal{C N}\left(0, \frac{1}{N} \mathbf{I}_{N}\right)$ with each entry mutually independent; further assuming $\mathbf{A}$ has uniformly bound spectral norm (with respect to $N$ ), then while $N$ converging to $\infty$, the following approximation equations can be established [6]

$$
\begin{aligned}
& \text { (i) } \mathbf{x}^{H} \mathbf{A} \mathbf{x}-\frac{1}{N} \operatorname{tr}(\mathbf{A}) \underset{N \rightarrow \infty}{\stackrel{\text { a.s. }}{\longrightarrow}} 0, \\
& \text { (ii) } \mathbf{x}^{H} \mathbf{A} \mathbf{x} \underset{N \rightarrow \infty}{\stackrel{\text { a.s. }}{\longrightarrow} 0 .}
\end{aligned}
$$

Lemma 3: Matrix inversion : For Hermitian invertible matrix $\mathbf{A} \in \mathbb{C}^{N \times N}$, scalar $\tau$, and vector $\mathbf{x} \in \mathbb{C}^{N}$, on condition that $\mathbf{A}+\tau \mathbf{x} \mathbf{x}^{H}$ is invertible, the following equality holds [6], [48]

$$
\frac{\mathbf{x}^{H} \mathbf{A}^{-1}}{1+\tau \mathbf{x}^{H} \mathbf{A}^{-1} \mathbf{x}}=\mathbf{x}^{H}\left(\mathbf{A}+\tau \mathbf{x} \mathbf{x}^{H}\right)^{-1} \text {. }
$$

${ }^{4}$ Here SIC is not considered in this SINR expression. The exact SINR with closed-form expression after SIC will be given by the following analysis. 
Corollary 1: With these two lemmas in hand, the prior SINR expression for user $k$ with equation (9) can be further written as

$$
\gamma_{k} \stackrel{\text { a.s. }}{\longrightarrow} \frac{1}{M} p_{k}\left|\mathbf{h}_{k}\right|^{2} \operatorname{tr}\left(\mathbf{W H P H} \mathbf{H}^{H} \mathbf{W}^{H}+\mathbf{R}_{n n}\right)^{-1} .
$$

Proof: Please see Appendix B.

Remark 1: The analytical processes from lemma 1 to corollary 1 are due to the application of lemma 3. Moreover, in the derivation procedure, it is assumed that with transmit and receive antenna growing, the received signal for user $k$ can be absorbed into the interferences from other users while calculating the $\gamma_{k}$.

Corollary 2: The $\mathbf{R}_{n n}$ can be given with a determinate equivalent expression as

$$
\mathbf{R}_{n n}=\sigma_{1}^{2} \mathbf{H}_{2} \mathbf{H}_{2}^{H}+\sigma_{2}^{2} \mathbf{I}_{K} \stackrel{\text { a.s. }}{\longrightarrow}\left(R \sigma_{1}^{2}+\sigma_{1}^{2}\right) \mathbf{I}_{K} .
$$

Proof: Please see Appendix C.

Remark 2: The deterministic equivalent with corollary 2 is due to the application of lemma 2. As shown here, the relay number becomes a constant coefficient of $\sigma_{1}$ in the nonregenerative massive-MIMO-NOMA relay systems.

\section{B. Achievable Capacity and Sum Rate of the Non-regenerative Massive-MIMO-NOMA Relay Systems}

The rough $\gamma_{k}$ expression with matrix variables is obtained by prior sub-section. Here in this sub-section, the purpose is to finally achieve the closed-form expression of $\gamma_{k}$. Afterwards, the capacity and sum rate performances can be easily addressed.

As proved by prior studies [6], [48], [49], in massive-MIMO system, with $K, M$ growing large, the empirical spectral distribution (ESD) of $\mathbf{W H}(\mathbf{W H})^{H}$ for all $K$ with expression

$$
\mu_{\frac{1}{\sqrt{M}}} \mathbf{W H}(\mathbf{W H})_{k}^{H}:=\frac{1}{M} \sum_{i=1}^{K} \frac{\delta_{\lambda_{i}}\left(\mathbf{W H}(\mathbf{W H})_{k}^{H}\right)}{\sqrt{K}},
$$

forms a tight sequence, where

$$
\lambda_{1}<\lambda_{2}<\ldots<\lambda_{K}
$$

are the eigenvalues of $\mathbf{W H}(\mathbf{W H})^{H}$, counting multiplicity. In addition, $\delta_{\lambda_{i}}$ denotes the Dirac measure with mass 1 concentrated in $i$. Under this condition, while further adopting the Stieltjes transform [48], [50] to $T_{\mathbf{W H}(\mathbf{W H})^{H}}\left(-\sigma^{2}\right)$, the following approximation holds

$$
T_{\mathbf{W H}(\mathbf{W H})^{H}}\left(-\sigma^{2}\right)-T_{K} \stackrel{a . s .}{\longrightarrow} 0,
$$

Similarly, the following equation also holds

$$
T_{\boldsymbol{H}(\boldsymbol{H})^{H}}-T_{M} \stackrel{\text { a.s. }}{\longrightarrow} 0 .
$$

Recall the prior studies in [51], [52], in Rayleigh fading channel assumption and additive white Gaussian noise (AWGN) that the noise are assumed to obey i.i.d. distribution with zero mean and same covariance between each channels both in the transmit to relay and relay to receiver system. The power gains are the exponential random variables [51]. With these assumptions, by applying the Marcenko-Pastur's
Theorem [52], with $K, M$ growing large, a determinate equivalent equation can be obtained while solving the (15) with corollary (2). Accordingly, based on the prior derivations and those introduced conclusions, the closed-form expression of $\gamma_{k}$ can be given by the following theorem.

Theorem 1: The SINR expressions of non-regenerative massive-MIMO-NOMA relay systems with closed-form expression: after the MMSE-SIC, SINR of each user $k$ can be given as

$$
\gamma_{k} \stackrel{a . s .}{\longrightarrow} \begin{cases}\frac{M p_{1}}{R^{2} \sigma_{1}^{2}+R \sigma_{2}^{2}}, & k=1, \\ \frac{p_{k}(-m+t)}{2\left(R \sigma_{1}^{2}+\sigma_{2}^{2}\right) M \sum_{j=2}^{k-1} p_{j}}, & 2 \leq k \leq K,\end{cases}
$$

with $t$

$$
t=\sqrt{m^{2}+4\left(R \sigma_{1}^{2}+\sigma_{2}^{2}\right) M^{2} \sum_{j=2}^{k-1} p_{j} \frac{M}{R}},
$$

and $m$

$$
m=\left(R \sigma_{1}^{2}+\sigma_{2}^{2}-\sum_{j=2}^{k-1} p_{j} \frac{M}{R}\right) M+\sum_{i=1}^{K} p_{i} \frac{M}{R} .
$$

Proof: Please see Appendix D.

Remark 3: The SINR expressions are achieved here so far. It can be found obviously from this expression that the noise power (with $\sigma_{1}^{2}, \sigma_{2}^{2}$ ) has a negative correlation to the $\gamma_{k}$.

Proposition 1: With the derived closed-form expression of $\gamma_{k}$, capacity of the non-regenerative massive-MIMO-NOMA relay systems will be

$$
C_{\text {sum }}=\sum_{k=2}^{K} \log \left(1+\gamma_{k}\right)+\log \left(1+\gamma_{1}\right),
$$

where $\gamma_{k}$ is given as the aforementioned solution by (21).

Proposition 2: While summing the achievable rate of each user with multiple sub-bands, the general closed-form achievable sum rate expression can be given as

$$
R_{\text {sum }}=S B\left\{\sum_{k=2}^{K} \log \left(1+\gamma_{k}\right)+\log \left(1+\gamma_{1}\right)\right\},
$$

Remark 4: As described by this closed-form sum rate expression, the allocated sub-band bandwidth and its number have positive correlations with the achievable sum rate, under a AWGN channel environment. The discussion on this obvious finding will be omitted in the following numerical simulations. In addition, compared with the prior work [3], [53], the new finding of this study is that with transceiver antenna number growing, the benefits of NOMA mainly lies in its multiplexing sub-band. In addition, the relay number together with the power value are additional dominant factors besides the massive-MIMO effect. The different power value for different user brings in a side effect to the system performance. This is because NOMA calling for rigorous discrimination among different users according to their allocated power values [19], [20].

The deduction accuracy of this study will be verified by the following numerical results. In addition, the non-intuitive effects of the transmit antenna number, relay number, receiver 
TABLE I

SIMULATION PARAMATERS

\begin{tabular}{|c|c|}
\hline Parameter & Value \\
\hline Monte Carlo simulations repeated & $10^{5}$ times \\
\hline Transmit antenna number $M$ & $100,200,300,400,500$ \\
\hline Receiver number $K$ & 10,20 \\
\hline Receiver to relay ratio $c_{2}$ & $\frac{1}{2}, \frac{1}{3}, \frac{1}{4}$ \\
\hline Sub-band number $S$ & 20 \\
\hline Sub-band width $B$ & $20 \mathrm{MHz}$ \\
\hline
\end{tabular}

number, NOMA allocated power to the system performance will be discussed as well by the numerical simulations.

\section{Numerical Results}

The analytical results are compared with the simulation results of the non-regenerative massive-MIMO-NOMA relay systems here in this section. In addition, the capacity, sum rate performances of the non-regenerative massive-MIMONOMA relay systems are investigated. Table I summarizes the simulation parameters that used in this section. Note that in the following simulation, for the sake of compactness, all of the power and noise values are set to be the normalized values with noise power as $1 \mathrm{~W}$.

The deduction of $\sigma_{1}^{2} \mathbf{H}_{2} \mathbf{H}_{2}^{H}$ with (16) is first verified via the Monte Carlo simulation beforehand with the normalized noise power value, whose results are shown by Fig. 4. In addition, $c_{2}$ value yields the user number divided by the relay number in this simulation. For example, given 10 user number, $c_{2}=1 / 2$ yields the relay number 20 . As shown, the analytical results match perfectly with the Monte Carlo simulation results. For instance, by 16 user number and $c_{2}=\frac{1}{4}$, the analytical result of $\sigma_{1}^{2} \mathbf{H}_{2} \mathbf{H}_{2}^{H}$ is 64 whereas the Monte Carlo simulation result is about 63.99. This demonstrates the correctness of the deduction here in this study. Additionally, observation from this figure has that noise power is further magnified by the increasing relay number. For instance, given 20 users in the non-regenerative massive-MIMO-NOMA relay systems, noise power is amplified $200 \%$ times from 40 to 80 relay numbers. The explanatory reason behind this is that, increasing transmit antenna number (relay number here) results in more degree of freedom (DoF) [22].

The $\gamma_{k}$ expression with theorem 1 are verified as well with the Monte Carlo simulation, where the parameters and their values are in tune with the simulation of Fig. 4. The simulation results are shown by Fig. 5. Observation clearly has that the analytical results with deterministic equivalent match closely with the simulation results. For instance, given 6 users, the analytical SINR is about $18.39 \mathrm{~dB}$ whereas the Monte Carlo based SINR is around $18.37 \mathrm{~dB}$, whose difference is about $0.02 \mathrm{~dB}$. Thus we can conclude that the analytical SINR with theorem 1 is correct. Furthermore, increasing the relay number will result in a decreasing SINR while keeping the other parameters constant. This is due to the increasing amplified noise power brought in by the growing relay number that confirmed by Fig. 4.

To verify the transmit antenna, relay as well as user number effects to the non-regenerative massive-MIMO-NOMA relay systems, the capacity and sum rate performances are

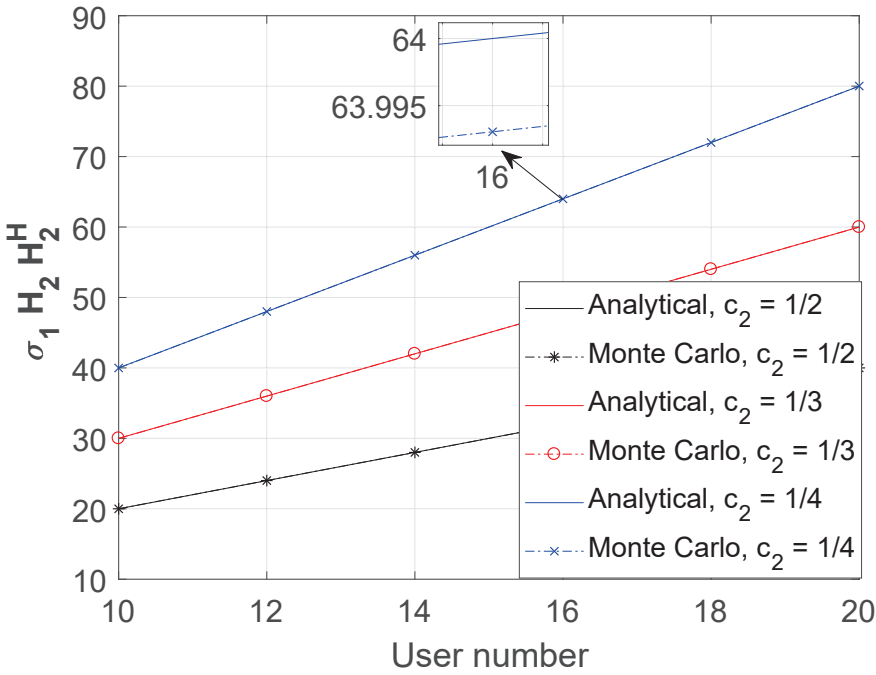

Fig. 4. The analytical and simulation results verification of $\sigma_{1}^{2} \mathbf{H}_{2} \mathbf{H}_{2}^{H}$ with normalized $\sigma_{1}$. The analytical results are calculated from (16).

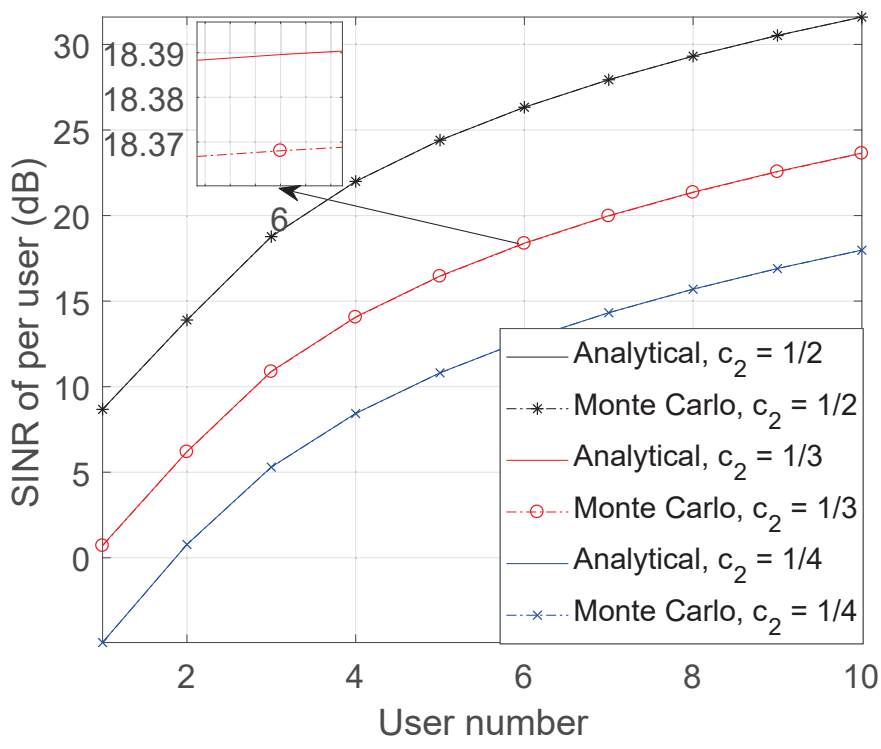

Fig. 5. The analytical and simulation results verification of $\gamma_{k}$ with $M=100$, normalized $\sigma_{1}$ and $\sigma_{2}$. The analytical results are calculated from (21).

compared. In this comparison, we assume two cases, with 10 user and with 20 user. The results are given by Fig. 6 and Fig. 7, according to (24) and (25). In these simulations, $c_{2}$ values are consist with prior simulations of Fig. 4. For instance, giving the user number $10, c_{2}=\frac{1}{2}, \frac{1}{3}, \frac{1}{4}$ yields the relay number 20,30,40. Similarly, given user number 20 , $c_{2}=\frac{1}{2}, \frac{1}{3}, \frac{1}{4}$ yields the relay number $40,60,80$. Moreover, $S=20$ and each sub-band with bandwidth value $20 \mathrm{MHz}$ are used for the sum rate calculation. By comparing with prior studies of relay assisted NOMA systems, the result in Fig. 6 reveals that the capacity performance of this non-regenerative massive-MIMO-NOMA relay systems surpass the prior relay assisted NOMA systems [37], [38] by one order of magnitude (10 times). In addition, by comparing with prior studies of MIMO-NOMA [25], [27], similar capacity enhancement can be witnessed as well. This is mainly due to the massive MIMO 


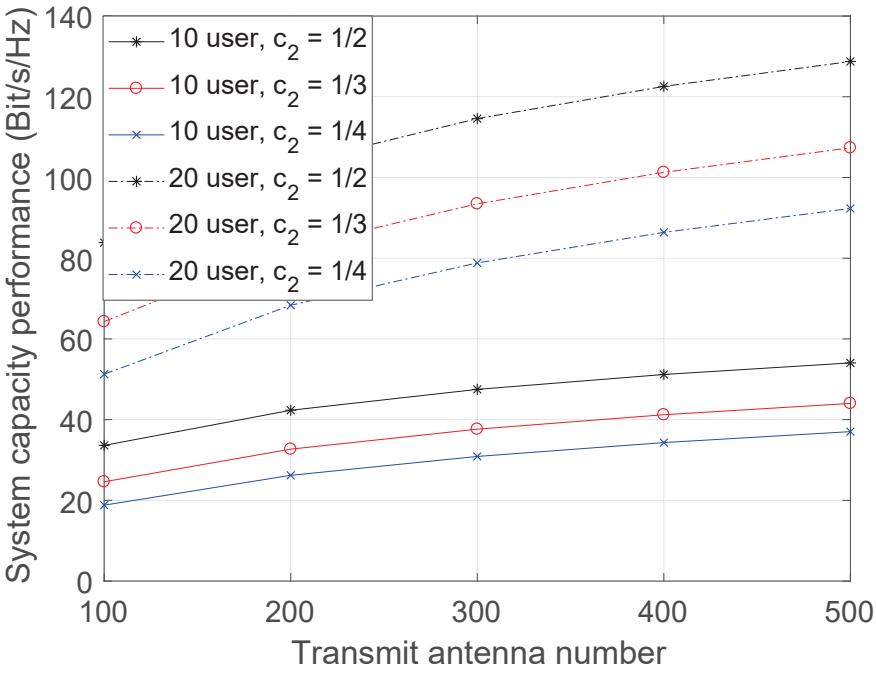

Fig. 6. The system capacity comparison while the number of transmitter, relay and user increasing. Two cases with user number 10 and 20 and normalized $\sigma_{1}$ and $\sigma_{2}$ are adopted. In addition, $C_{2}=\frac{1}{2}, \frac{1}{3}, \frac{1}{4}$ yields the relay number $20,30,40$ with 10 users; and 40,60, 80 with 20 users. The analytical results are calculated from (24) with (21).

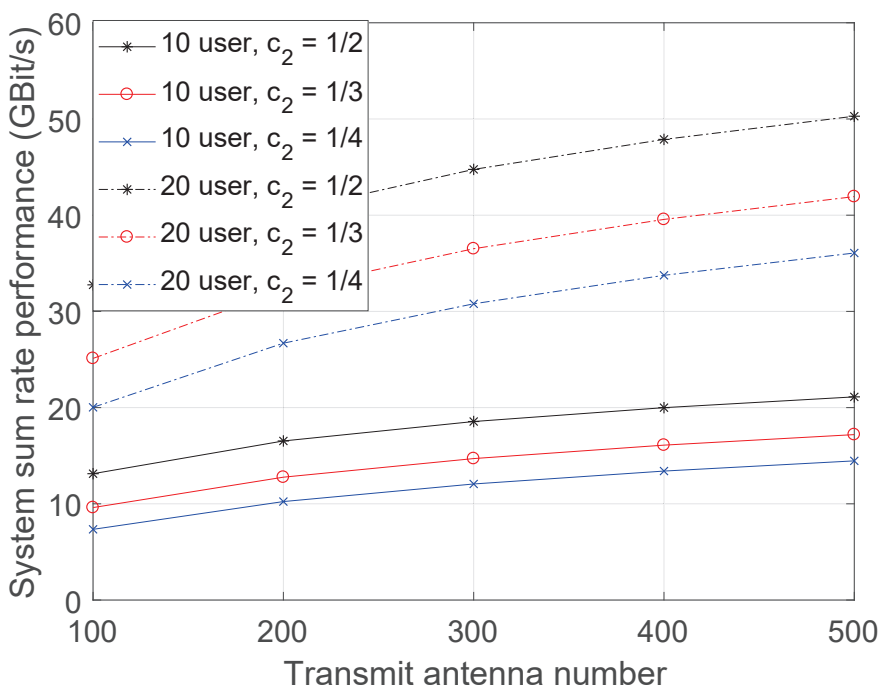

Fig. 7. The system sum rate comparison while the number of transmitter, relay and user increasing. Two cases with user number 10 and 20 and normalized $\sigma_{1}$ and $\sigma_{2}, S=20, B=20 \mathrm{MHz}$ are adopted. In addition, $C_{2}=\frac{1}{2}, \frac{1}{3}, \frac{1}{4}$ yields the relay number $20,30,40$ with 10 users; and $40,60,80$ with 20 users. The analytical results are calculated from (25) with (21).

effects. By given the same bandwidth, it is divinable that the sum rate will be one order of magnitude as well.

Several other results can be gained from Fig. 6 and Fig. 7. Firstly, growing relay number always results in inferior capacity and sum rate performances, which is mainly due to the increasing amplified channel noise in the first relay phase that verified from Fig. 4. Secondly, increasing transmit antenna number displays a decreasing positive effect to the capacity and sum rate performances given the constant relay and user numbers. The explanatory reason is due to the Shannon theory with (24). With this in hand, the qualitative explanation of the performance with Fig. 6 and Fig. 7 is to proof (21) yields an incremental reduction while $M$ growing large, which is given by the Appendix E. Additionally, growing user number will lead to better capacity and sum rate performances. While further combining the results here with Fig. 5, it is also noticed that the user number's positive effect to the capacity and sum rate performances become weak while linearly growing. This is due to the increasing NOMA co-channel interferences from other users bring in inferior $\gamma_{k}$ value, which in turn affect the capacity and sum rate performances.

The system capacity performance comparison between the proposed massive-MIMO-NOMA and the massive-MIMOOMA are addressed within the non-regenerative relay system. In line with prior studies [54], with MMSE, the SINR expression of each user $k$ with massive-MIMO in the nonregenerative relay assisted systems can be given as

$$
\gamma_{k, O M A} \stackrel{\text { a.s. }}{\longrightarrow} \frac{M P_{k}}{R^{2} \sigma_{1}^{2}+R \sigma_{2}^{2}} .
$$

Proof: Please see Appendix F.

With the addressed $\gamma_{k, O M A}$, the capacity performance can be easily obtained according to the Shannon theory, as discussed before by (10). The capacity performance comparison of massive-MIMO-NOMA and massive-MIMO-OMA, within the non-regenerative relay systems, is shown by Fig. 8. Here in massive-MIMO-NOMA scenario, each bandwidth is allocated 10 users as an example. The achievable capacity is averaged over 10 users accordingly. As shown, each massive-MIMONOMA user brings about $30 \% \sim 50 \%$ capacity increment compared to massive-MIMO-OMA scenario. For instance, by $c_{2}=\frac{1}{3}$ and 100 transmit antenna, the corresponding capacity are $7.78 \mathrm{Bit} / \mathrm{s} / \mathrm{Hz} / \mathrm{user}$ and $5.91 \mathrm{Bit} / \mathrm{s} / \mathrm{Hz} / \mathrm{user}$ with massive-MIMO-NOMA and massive-MIMO-OMA scenarios. The massive-MIMO-NOMA capacity increment of each user is about $31.64 \%$ compared to massive-MIMO-OMA scenario. In addition, as shown by this figure, the massive-MIMONOMA capacity merit is even better while transmit antenna number growing large. This is because that more DoF brings in even higher channel SNR for each user [3]. This better channel SNR further strengthens the merit of massive-MIMO-NOMA compared to the massive-MIMO-OMA scenario.

Fig. 9 and Fig. 10 further check the growing SNR effects to the capacity and sum rate performances. Similarly, this is done according to (24) and (25). Without loss of generality, here in this simulation, the vertical line with SNR is averaged over all 10 users, which means,

$$
\mathrm{SNR}=\frac{\sum_{i=1}^{K} p_{i}}{K\left(R \sigma_{1}^{2}+\sigma_{2}^{2}\right)},
$$

where $\sigma_{1}^{2}, \sigma_{2}^{2}$ values are normalized. Firstly, the prior finding with Fig. 6 and Fig. 7 that the decreasing positive effect to the capacity and sum rate performances of the increasing transmit antenna number is further verified, as clearly shown by this figure. Additionally, while keeping other factors remain, better capacity and sum rate can always be achieved by increasing the SNR. This is another evidence of the deduction accuracy in this study, according to the prior conclusion with the studies in [6], [22]. 


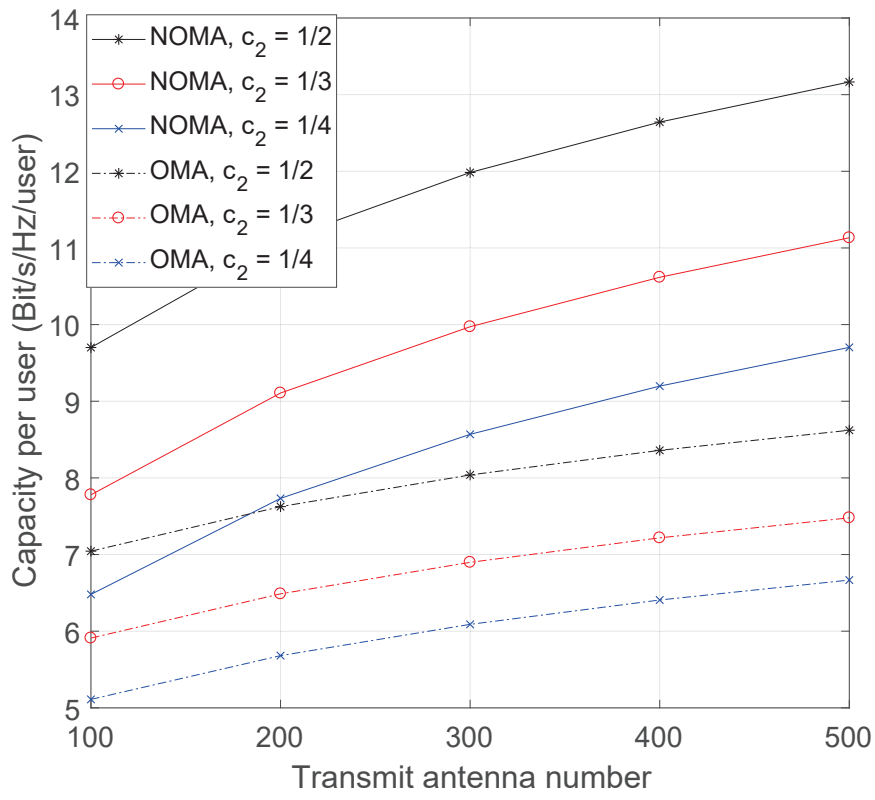

Fig. 8. System capacity performance comparison between NOMA and OMA mechanism with normalized $\sigma_{1}$ and $\sigma_{2}$. The analytical results of massiveMIMO-NOMA is calculated from (24) with (21). The analytical results of massive-MIMO-OMA is calculated from Shannon capacity theory with (26).

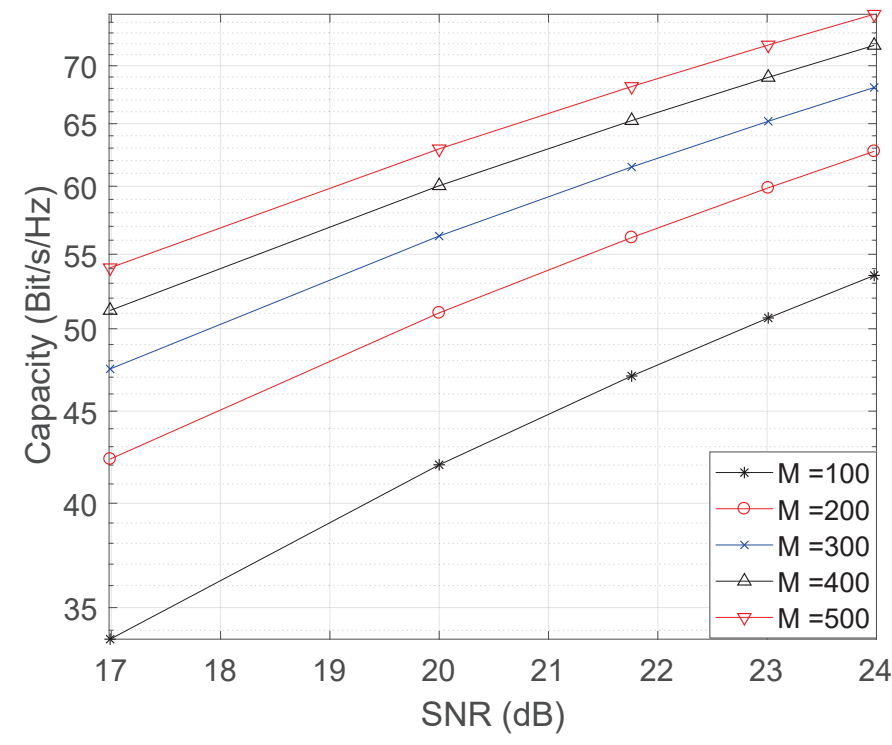

Fig. 9. Capacity performance of 10 users while SNR growing with normalized $\sigma_{1}$ and $\sigma_{2}$. The analytical results of massive-MIMO-NOMA is calculated from (24) with (21).

\section{Conclusion}

The non-regenerative massive-MIMO-NOMA relay systems were introduced here to enhance the system SE and further enlarge the coverage range for 5G. Moreover, the MMSE-SIC decoding method was adopted to decode the received information. The system capacity and sum rate were addressed by the closed-form expressions with the help of matrix theories. It was demonstrated that the transmit antenna number and user number performing the positive but decreasing correlations to the system capacity and sum rate performances, which is different from prior sole massive-MIMO system. In addition,

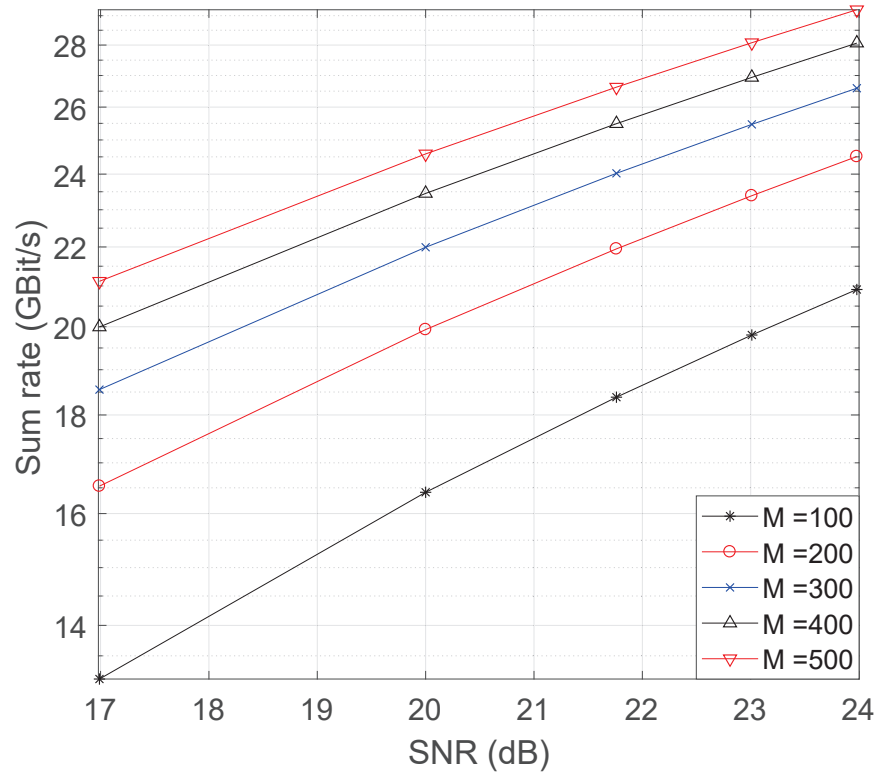

Fig. 10. Sum rate performance of 10 users while SNR growing with normalized $\sigma_{1}$ and $\sigma_{2}, S=20, B=20 \mathrm{MHz}$. The analytical results are calculated from (25) with (21).

the increasing averaged SNR value always brings in better capacity and sum rate performances. Compared with massiveMIMO-OMA regime, the massive-MIMO-NOMA regime can achieve better system performance. This merit becomes even better with transmit antenna number growing large. The NOMA user pair problem with different quality of service (QoS) requirements can be further investigated in future with the non-regenerative massive-MIMO-NOMA relay systems.

\section{APPENDIX A}

Proof: As discussed before, by following the prior deduction in [6], [48], [55], while decoding the received information, MMSE is the optimal solution especially in the massiveMIMO systems [6], [47]. With this conclusion in hand, while adopting MMSE at the receiver side and multiplying it by (5), within each sub-band, the following equality holds

$\operatorname{MMSE}_{k} / S=\frac{\sqrt{P_{k}} \mathbf{h}_{k}^{H} \mathbf{w}_{k}^{H}\left(\mathbf{W H P H} \mathbf{H}^{H} \mathbf{W}^{H}+\mathbf{R}_{n n}\right)^{-1} \mathbf{y}_{k}}{S}$,

where $\mathbf{h}_{k}, \mathbf{W}_{k}$ denote the channel and pre-coder for $k$ th user. In addition, with the non-regenerative massive-MIMO-NOMA relay systems, observation has that

$$
\boldsymbol{R}_{n n}=\sigma_{1}^{2} \mathbf{H}_{1} \mathbf{H}_{1}^{H}+\sigma_{2}^{2} \mathbf{I}_{K},
$$

where $\sigma_{1}^{2}, \sigma_{2}^{2}$ are the covariance of equivalent noise power matrix with $\mathbf{n}_{1} \mathbf{n}_{1}^{H}, \mathbf{n}_{2} \mathbf{n}_{2}^{H}$. This gives the received signal power of $k$ th user by

$$
P_{k}=\left[p_{k}\left|\mathbf{h}_{k}\right|^{2} \mathbf{w}_{k}^{H}\left(\sum_{i=1}^{K} p_{i}\left|\mathbf{h}_{i}\right|^{2} \mathbf{w}_{i} \mathbf{w}_{i}^{H}+\mathbf{R}_{n n}\right)^{-1} \mathbf{w}_{k}\right]^{2} .
$$

Here $\mathbf{W}_{i}$ is the optimal MMSE receiver. In this case, before adopting the SIC to eliminate the co-channel interferences, the power of interference plus noise can be given as (32). 


$$
\begin{aligned}
P_{I R} & =p_{k}\left|\mathbf{h}_{k}\right|^{2} \sum_{i=1, i \neq k}^{K} p_{i}\left|\mathbf{h}_{i}\right|^{2}\left|\mathbf{w}_{k}^{H}\left(\sum_{i=1}^{K} P_{j}\left|\mathbf{h}_{j}\right|^{2} \mathbf{w}_{j} \mathbf{w}_{j}^{H}+\mathbf{R}_{n n}\right)^{-1} \mathbf{w}_{k}\right|^{2}+\mathbf{R}_{n n} p_{k}\left|\mathbf{h}_{k}\right|^{2} \mathbf{w}_{k}^{H}\left(\sum_{i=1}^{K} p_{i}\left|\mathbf{h}_{j}\right|^{2} \mathbf{w}_{j} \mathbf{w}_{j}^{H}+\mathbf{R}_{n n}\right)^{-2} \mathbf{w}_{k} \\
& \stackrel{a}{=} p_{k}\left|\mathbf{h}_{k}\right|^{2} \mathbf{w}_{k}^{H}\left(\sum_{i=1}^{K} p_{i}\left|\mathbf{h}_{i}\right|^{2} \mathbf{w}_{i} \mathbf{w}_{i}^{H}+\mathbf{R}_{n n}\right)^{-1} \mathbf{w}_{k}-\left[p_{k}\left|\mathbf{h}_{k}\right|^{2} \mathbf{w}_{k}^{H}\left(\sum_{i=k}^{K} p_{i}\left|\mathbf{h}_{k}\right|^{2} \mathbf{w}_{i} \mathbf{w}_{i}^{H}+\mathbf{R}_{n n}\right)^{-1} \mathbf{w}_{k}\right]^{2} .
\end{aligned}
$$

For the sake of explanation convenience, here in (32), the equality's left side of its first part denoting the interferences from other users, and the second part is the noise power, where the equality of $\stackrel{a}{=}$ is due to the fact that

$$
\begin{aligned}
& \sum_{i=1, i \neq k}^{K} p_{i}\left|\mathbf{h}_{i}\right|^{2} \mathbf{W}_{i} \mathbf{W}_{i}^{H}=\left(\sum_{i=1}^{K} p_{i}\left|\mathbf{h}_{i}\right|^{2} \mathbf{W}_{i} \mathbf{W}_{i}^{H}+\mathbf{R}_{n n}\right) \\
& -p_{k}\left|\mathbf{h}_{k}\right|^{2} \mathbf{w}_{k} \mathbf{w}_{k}^{H}-\mathbf{R}_{n n} .
\end{aligned}
$$

Consequently, by given the signal power as (30) and interference plus noise power as (32), the SINR of user $k$ will turn out to be

$$
\gamma_{k}=\frac{\left[p_{k}\left|\mathbf{h}_{k}\right|^{2} \mathbf{w}_{k}^{H}\left(\sum_{i=1}^{K} p_{i}\left|\mathbf{h}_{i}\right|^{2} \mathbf{w}_{i} \mathbf{w}_{i}^{H}+\mathbf{R}_{n n}\right)^{-1} \mathbf{w}_{k}\right]^{2}}{P_{I R}} .
$$

Additionally, by further eliminating the common factor of both numerator and denominator, we can finally arrive at the following equality

$$
\gamma_{k}=\frac{p_{k}\left|\mathbf{h}_{k}\right|^{2} \mathbf{w}_{k}^{H}\left(\sum_{i=1}^{K} p_{i}\left|\mathbf{h}_{k}\right|^{2} \mathbf{w}_{i} \mathbf{w}_{i}^{H}+\mathbf{R}_{n n}\right)^{-1} \mathbf{w}_{k}}{1-p_{k}\left|\mathbf{h}_{k}\right|^{2} \mathbf{w}_{k}^{H}\left(\sum_{i=1}^{K} p_{i}\left|\mathbf{h}_{i}\right|^{2} \mathbf{w}_{i} \mathbf{w}_{i}^{H}+\mathbf{R}_{n n}\right)^{-1} \mathbf{w}_{k}} .
$$

This completes the proof.

\section{APPENDIX B}

Proof: While applying the conclusion of Lemma 3 to Lemma 1, (9) can be rewritten as

$$
\gamma_{k}=p_{k}\left|\mathbf{h}_{k}\right|^{2} \mathbf{w}_{k}^{H}\left(\sum_{i=1, i \neq k}^{K} p_{i}\left|\mathbf{h}_{i}\right|^{2} \mathbf{w}_{i} \mathbf{w}_{i}^{H}+\mathbf{R}_{n n}\right)^{-1} \mathbf{w}_{k} .
$$

Further adopting the Lemma 2, as $K, M$ growing large with a ratio $c=K / M$, whereas the constraints $0<\liminf _{M} c \leq$ $\lim \sup _{M} \frac{1}{c}<\infty$ holds $^{5}$, SINR in this case, will follow

$$
\begin{aligned}
\gamma_{k} & =\frac{1}{M} p_{k}\left|\mathbf{h}_{k}\right|^{2} \operatorname{tr}\left(\sum_{i=1, i \neq k}^{K} p_{i}\left|\mathbf{h}_{i}\right|^{2} \mathbf{w}_{i} \mathbf{w}_{i}^{H}+\mathbf{R}_{n n}\right)^{-1} \\
& \stackrel{a . s .}{\longrightarrow} \frac{1}{M} p_{k}\left|\mathbf{h}_{k}\right|^{2} \operatorname{tr}\left(\sum_{i=1, i \neq k}^{S} p_{i} \mathbf{W} \mathbf{H} \mathbf{H}^{\mathbf{H}} \mathbf{W}^{\mathbf{H}}\right. \\
& \left.+p_{k} \mathbf{W} \mathbf{H} \mathbf{H}^{\mathbf{H}} \mathbf{W}^{\mathbf{H}}+\mathbf{R}_{n n}\right)^{-1} \\
& =\frac{1}{M} p_{k}\left|\mathbf{h}_{k}\right|^{2} \operatorname{tr}\left(\mathbf{W} \mathbf{H} \mathbf{P} \mathbf{H}^{H} \mathbf{W}^{H}+\mathbf{R}_{n n}\right)^{-1}
\end{aligned}
$$

This completes the proof.

\footnotetext{
${ }^{5}$ This hypothesis is to claim that $\mathrm{c}$ is a constant number within region $[0, \infty]$, where the value of $\mathrm{c}$ is no less than the infimum value and no greater than the supremum value with regards to $M$.
}

\section{APPENDIX C}

Proof: In this proof, lemma 2 is invoked to achieve a closed-form expression of the following equality

$$
\mathbf{R}_{n n}=\sigma_{1}^{2} \mathbf{H}_{2} \mathbf{H}_{2}^{H}+\sigma_{2}^{2} \mathbf{I}_{K} .
$$

Firstly, observation has that the first part of the equation can be decomposed as

$$
\sigma_{1}^{2} \mathbf{H}_{2} \mathbf{H}_{2}^{H}=\sigma_{1}^{2} \sum_{j=1}^{R} \mathbf{h}_{2, j} \mathbf{h}_{2, j}^{H}=\sigma_{1}^{2} \sum_{j=1}^{R} \mathbf{h}_{2, j} \mathbf{I}_{K} \mathbf{h}_{2, j}^{H} .
$$

Thus by following lemma 2 , for each $1 \leq j \leq R$, the following equation holds

$$
\mathbf{h}_{2, j} \mathbf{I}_{K} \mathbf{h}_{2, j}^{H}-\frac{1}{K} \operatorname{tr}\left(\mathbf{I}_{K}\right) \stackrel{\text { a.s. }}{\longrightarrow} 0 .
$$

Then we can arrive the following deterministic equivalent of $\sigma_{1}^{2} \mathbf{H}_{2} \mathbf{H}_{2}^{H}$

$$
\sigma_{1}^{2} \mathbf{H}_{2} \mathbf{H}_{2}^{H}-\left(\frac{R}{K} \sigma_{1}^{2} \operatorname{tr}\left(\mathbf{I}_{K}\right)+\sigma_{1}^{2} \mathbf{I}_{K}\right) \stackrel{\text { a.s. }}{\longrightarrow} 0 .
$$

This means, the deterministic equivalent of $\sigma_{1}^{2} \mathbf{H}_{2} \mathbf{H}_{2}^{H}+\sigma_{2}^{2} \mathbf{I}_{K}$ is

$$
\sigma_{1}^{2} \mathbf{H}_{2} \mathbf{H}_{2}^{H}+\sigma_{2}^{2} \mathbf{I}_{K} \stackrel{\text { a.s. }}{\longrightarrow}\left(R \sigma_{1}^{2}+\sigma_{1}^{2}\right) \mathbf{I}_{K} .
$$

This completes the proof.

\section{APPENDIX D}

Proof: In this proof, the derivations are divided into two cases: $k=1$ and $2<k<K$. This is due to the fact that while decoding the transmit information with SIC, for $k=1$, all of the co-channel interferences has already been perfectly eliminated; whereas for $2<k<K$, the co-channel interference from other user with a lower order cannot be eliminated with SIC.

Firstly, in line with the prior proof of Appendix C, the determinant equivalent of SINR has the following approximation

$$
\begin{aligned}
& \frac{1}{M} p_{k}\left|\mathbf{h}_{k}\right|^{2} \operatorname{tr}\left(\mathbf{W H P H} \mathbf{H}^{H} \mathbf{W}^{H}+\mathbf{R}_{n n}\right)^{-1} \\
& \stackrel{a . s .}{\longrightarrow} \frac{1}{M} p_{k}\left|\mathbf{h}_{k}\right|^{2} \operatorname{tr}\left(\mathbf{W H P H} \mathbf{H}^{H} \mathbf{W}^{H}+\left(R \sigma_{1}^{2}+\sigma_{1}^{2}\right) \mathbf{I}_{K}\right)^{-1} .
\end{aligned}
$$

In addition, applying the Stieltjes transform [48], [50] to $\mathbf{W H P H} \mathbf{H}^{H} \mathbf{W}^{H}$ yields the following expression

$$
S_{\mathbf{W H P H}^{H} \mathbf{W}^{H}}(z)-S_{M}(z) \stackrel{\text { a.s. }}{\longrightarrow} 0 .
$$


For user number $2 \leq k \leq K$, by applying the SIC to eliminate the co-channel interferences with higher power values, $S_{M}(z)$ denoting the unique positive solution of

$$
s=\left[\frac{1}{M} \frac{\sum_{i=i}^{K} p_{i}|\mathbf{h}|_{i}^{2}}{1+s \sum_{j=2}^{k-1} p_{j}|\mathbf{h}|_{j}^{2}}+\left(R \sigma_{1}^{2}+\sigma_{1}^{2}\right) \mathbf{I}_{K}\right]^{-1} .
$$

Here $j \leq k-1$ is due to fact that SIC can successfully eliminate the co-channel interferences from other users with higher user orders. Thus by solving the equation with (45), an unique positive solution can be given as

$$
s=\frac{-m+t}{2\left(R \sigma_{1}^{2}+\sigma_{2}^{2}\right) M \sum_{j=2}^{k-1} p_{j}\left|\mathbf{h}_{j}\right|^{2}},
$$

where $t$ is

$$
t=\sqrt{m^{2}+4\left(R \sigma_{1}^{2}+\sigma_{2}^{2}\right) M^{2} \sum_{j=2}^{k-1} p_{j}\left|\mathbf{h}_{j}\right|^{2}},
$$

with $m$ given as

$$
m=\left(R \sigma_{1}^{2}+\sigma_{2}^{2}-\sum_{j=2}^{k-1} p_{j}\left|\mathbf{h}_{j}\right|^{2}\right) M+\sum_{i=1}^{K} p_{i}\left|\mathbf{h}_{i}\right|^{2} .
$$

To further eliminate the channel uncertainty with the deterministic equivalent tools in order to get a closed-form expression of the $\gamma_{k}$ when $2 \leq k \leq K$, as discussed aforementioned, the AWGN channel assumption is assumed in line with the assumption in lemma 2 for both the first and second part of the non-regenerative massive-MIMO-NOMA relay systems. Moreover, it is noticed that with this assumption, for each user $k$, the following equivalent holds

$$
|\mathbf{h}|_{k}^{2}=\mathbf{H}_{1} \mathbf{h}_{2, k} \mathbf{h}_{2, k}^{H} \mathbf{H}_{1}^{H}=\sum_{i=1}^{M} \mathbf{h}_{1, i} \mathbf{h}_{2, k} \mathbf{h}_{2, k}^{H} \mathbf{h}_{1, i}^{H} .
$$

Substituting the conclusion of lemma 2 into the above equation, it has

$$
|\mathbf{h}|_{k}^{2}-\frac{M}{R} \operatorname{tr}\left(\mathbf{h}_{2, k} \mathbf{h}_{2, k}^{H}\right) \stackrel{\text { a.s. }}{\longrightarrow} 0 .
$$

Additionally, it is noticed that $\mathbf{h}_{2, k} \mathbf{h}_{2, k}^{H}$ can be further written as

$$
\mathbf{h}_{2, k} \mathbf{h}_{2, k}^{H}=\mathbf{h}_{2, k} \mathbf{I}_{K} \mathbf{h}_{2, k}^{H} .
$$

Thus by further employing the processing method with appendix $\mathbf{C}$, the final deterministic equivalent of $|\mathbf{h}|_{k}^{2}$ can be given as

$$
|\mathbf{h}|_{k}^{2}-\frac{M}{R} \stackrel{\text { a.s. }}{\longrightarrow} 0 .
$$

Consequently, by substituting this result into (46) with (47) and (48), and further substituting the results into (37), one can finally obtain the closed-form expression of the $\gamma_{k}$ with $2 \leq k \leq K$

$$
\gamma_{k} \stackrel{a . s .}{\longrightarrow} \frac{p_{k}(-m+t)}{2\left(R \sigma_{1}^{2}+\sigma_{2}^{2}\right) M \sum_{j=2}^{k-1} p_{j}},
$$

where $t$ is

$$
t=\sqrt{m^{2}+4\left(R \sigma_{1}^{2}+\sigma_{2}^{2}\right) M^{2} \sum_{j=2}^{k-1} p_{j} \frac{M}{R}},
$$

with $m$ given as

$$
m=\left(R \sigma_{1}^{2}+\sigma_{2}^{2}-\sum_{j=2}^{k-1} p_{j} \frac{M}{R}\right) M+\sum_{i=1}^{K} p_{i} \frac{M}{R} .
$$

Other than $2<k<K$, the closed-form expression when $k=1$ can be straightforwardly obtained. That is, for $k=1$, it is noticed that the co-channel interferences from other users with NOMA scheme have been perfectly eliminated with SIC. This yields the $\gamma_{1}$ with following expression

$$
\gamma_{1}=\frac{p_{1}\left|\mathbf{h}_{1}\right|^{2}}{R \sigma_{1}^{2}+\sigma_{2}^{2}} \stackrel{a . s .}{\longrightarrow} \frac{M p_{1}}{R^{2} \sigma_{1}^{2}+R \sigma_{2}^{2}} .
$$

Thus combining (53) and (56) will be the SINR expression of each user in the non-regenerative massive-MIMO-NOMA relay systems.

This completes the proof.

\section{APPENDIX E}

Proof: The qualitative analysis of capacity and sum rate performances with $M$ growing large is addressed here in this section. Primarily, observation has that for each user $k$, on condition that $M$ is larger than $R$ and $K$, while merely growing $M$ and keeping $R$ and $K$ remain, the values of $\sum_{j=1}^{k-1} p_{j}\left|\mathbf{h}_{j}\right|^{2}, \sum_{i=1}^{K} p_{i}\left|\mathbf{h}_{i}\right|^{2}, p_{k}\left|\mathbf{h}_{k}\right|^{2}$ can be taken as constant values. In addition, for the sake of analysis compactness, following assumptions are given beforehand: $a=\left(R \sigma_{1}^{2}+\sigma_{2}^{2}-\sum_{j=2}^{k-1} p_{j} \frac{M}{R}\right), b=\sum_{i=1}^{K} p_{i} \frac{M}{R}, c=$ $2\left(R \sigma_{1}^{2}+\sigma_{2}^{2}\right) M^{2} \sum_{j=2}^{k-1} p_{j} \frac{M}{R}, d=p_{k} \frac{M}{R}$. In this case, $\gamma_{k}$ can be rewritten as

$$
\gamma_{k}=\frac{d\left[-(a M+b)+\sqrt{(a M+b)^{2}+2 c M^{2}}\right]}{c M} .
$$

It is noticed that $a, b, c, d$ turn out to be constant elements when all factors are given in the non-regenerative massive-MIMONOMA relay systems. In this case, the following proportional holds

$$
\gamma_{k} \propto \frac{-(a M+b)+\sqrt{(a M+b)^{2}+2 c M^{2}}}{c M} .
$$

Furthermore, dividing the numerator and the denominator with $M$ yields

$$
\gamma_{k} \propto \frac{-a+\left\{-\frac{b}{M}+\sqrt{(a+2 c)+O\left(\left(\frac{b}{M}\right)^{2}\right)+\left(\frac{b}{M}\right)^{2}}\right\}}{c} .
$$

It is clearly shown that while keeping other elements constant, $O\left(\left(\frac{b}{M}\right)^{2}\right)$ determines the differential gap between $-\frac{b}{M}$ and $\sqrt{(a+2 c)+O\left(\left(\frac{b}{M}\right)^{2}\right)+\left(\frac{b}{M}\right)^{2}}$. Moreover, with $M$ growing large, the gap becomes smaller, which leads to a smaller increment of $\gamma_{k}$. This finally brings in smaller increment of capacity and sum rate.

This completes the proof. 


\section{APPENDIX F}

Proof: In line with prior study of [54], with MMSE massive-MIMO, the SINR expression of user $k$ can be given as

$$
\gamma_{k, O M A}=\frac{1}{\left(\mathbf{I}+\rho|\mathbf{h}|_{k}^{2}\right)^{-1}}-1 .
$$

where $\rho$ yields the SNR of each user. Within the nonregenerative massive-MIMO-OMA relay systems, by applying the prior derived conclusions of Appendix $\mathrm{C}, \rho$ can be rewritten as

$$
\rho=\frac{P_{k}}{R \sigma_{1}^{2}+\sigma_{2}^{2}} .
$$

Additionally, with Appendix D, the following approximation of this non-regenerative massive-MIMO-OMA relay systems holds

$$
|\mathbf{h}|_{k}^{2} \stackrel{\text { a.s. }}{\longrightarrow} \frac{M}{R} .
$$

While subscribing (61) and (62) into (60), we can safely arrive at the conclusion.

This completes the proof.

\section{REFERENCES}

[1] D. Zhang, Z. Zhou, S. Mumtaz, J. Rodriguez, and T. Sato, "One integrated energy efficiency proposal for 5G IoT communications," IEEE Internet Things J., vol. 3, no. 6, pp. 1346-1354, Dec. 2016.

[2] W. Liu, S. Han, C. Yang, and C. Sun, "Massive MIMO or small cell network: Who is more energy efficient?" in IEEE WCNC workshops, Apr. 2013, pp. 24-29.

[3] F. Rusek, D. Persson, B. K. Lau, E. G. Larsson, T. L. Marzetta O. Edfors, and F. Tufvesson, "Scaling up MIMO: Opportunities and challenges with very large arrays," IEEE Signal Processing Mag., vol. 30, no. 1, pp. 40-60, Jan. 2013.

[4] H. Q. Ngo, E. G. Larsson, and T. L. Marzetta, "Energy and spectral efficiency of very large multiuser MIMO systems," IEEE Trans. Commun., vol. 61, no. 4, pp. 1436-1449, Apr. 2013.

[5] H. Beyranvand, M. Levesque, M. Maier, J. A. Salehi, C. Verikoukis, and D. Tipper, "Toward 5G: FiWi enhanced LTE-A hetnets with reliable lowlatency fiber backhaul sharing and WiFi offloading," IEEE/ACM Trans. Netw., vol. PP, no. 99, pp. 1-18, Sep. 2016.

[6] J. Hoydis, S. Ten Brink, and M. Debbah, "Massive MIMO in the UL/DL of cellular networks: How many antennas do we need?" IEEE J. Sel. Areas Commun., vol. 31, no. 2, pp. 160-171, Feb. 2013.

[7] D. Zhang, M. Tariq, S. Mumtaz, J. Rodriguez, and T. Sato, "Integrating energy efficiency analysis of massive MIMO based C-RAN," EURASIP J. Wirel. Commun. Netw., vol. 2016, no. 1, pp. 277-286, Dec. 2016.

[8] L. Dai, B. Wang, Y. Yuan, S. Han, C. 1. I, and Z. Wang, "Non-orthogonal multiple access for 5G: Solutions, challenges, opportunities, and future research trends," IEEE Commun. Mag., vol. 53, no. 9, pp. 74-81, Sep. 2015.

[9] P. Wang, J. Xiao, and L. P, "Comparison of orthogonal and nonorthogonal approaches to future wireless cellular systems," IEEE Veh. Technol. Mag., vol. 1, no. 3, pp. 4-11, Sep. 2006.

[10] Y. Cai, Z. Qin, F. Cui, G. Y. Li, and J. A. McCann, "Modulation and multiple access for 5g networks," CoRR, vol. abs/1702.07673. [Online]. Available: https://arxiv.org/abs/1702.07673, 2017.

[11] P. Parida and S. S. Das, "Power allocation in OFDM based NOMA systems, a DC programming approach," in IEEE Globecom, Dec. 2014, pp. 1026-1031.

[12] H. Nikopour and H. Baligh, "Sparse code multiple access," in IEEE PIMRC, Sep. 2013, pp. 332-336.

[13] J. Zeng, B. Li, X. Su, L. Rong, and R. Xing, "Pattern division multiple access (PDMA) for cellular future radio access," in IEEE WCSP, Oct. 2015, pp. 1-5.

[14] Y. Saito, Y. Kishiyama, A. Benjebbour, T. Nakamura, A. Li, and K. Higuchi, "Non-orthogonal multiple access (NOMA) for cellular future radio access," in IEEE VTC Spring, June 2013, pp. 1-5.
[15] Z. Ding, Z. Yang, P. Fan, and H. Poor, "On the performance of nonorthogonal multiple access in $5 \mathrm{G}$ systems with randomly deployed users," IEEE Signal Process. Lett., vol. 21, no. 12, pp. 1501-1505, Dec. 2014.

[16] Z. Ding, M. Peng, and H. Poor, "Cooperative non-orthogonal multiple access in 5G systems," IEEE Commun. Lett., vol. 19, no. 8, pp. 1462 1465, Aug 2015.

[17] Z. Ding, P. Fan, and H. V. Poor, "Impact of user pairing on 5G nonorthogonal multiple-access downlink transmissions," IEEE Trans. Vehi. Tech., vol. 65, no. 8, pp. 6010-6023, Aug. 2016.

[18] Z. Ding, Y. Liu, J. Choi, Q. Sun, M. Elkashlan, C.-L. I, and H. V. Poor, "Application of non-orthogonal multiple access in LTE and 5G networks," IEEE Commun. Mag., vol. 55, no. 2, pp. 185-191, Feb. 2017.

[19] J. Choi, "H-ARQ based non-orthogonal multiple access with successive interference cancellation," in IEEE Globecom, Nov. 2008, pp. 1-5.

[20] _ "On the power allocation for MIMO-NOMA systems with layered transmissions," IEEE Trans. Wireless Commun., vol. 15, no. 5, pp. 32263237, May 2016.

[21] Z. Ding, F. Adachi, and H. V. Poor, "The application of MIMO to nonorthogonal multiple access," IEEE Trans. Wireless Commun., vol. 15 no. 1 , pp. 537-552, Jan. 2016.

[22] D. Tse and P. Viswanath, Fundamentals of Wireless Communication Cambridge, UK: Cambridge University Press, 2005.

[23] Y. Liu, Z. Ding, M. Elkashlan, and J. Yuan, "Non-orthogonal multiple access in large-scale underlay cognitive radio networks," IEEE Trans. Vehi. Tech., vol. PP, no. 99, pp. 1-6, Feb. 2016.

[24] Y. Liu, Z. Ding, M. Elkashlan, and H. V. Poor, "Cooperative nonorthogonal multiple access with simultaneous wireless information and power transfer," IEEE J. Sel. Areas Commun., vol. 34, no. 4, pp. 938 953, Apr. 2016

[25] Y. Liu, G. Pan, H. Zhang, and M. Song, "On the capacity comparison between MIMO-NOMA and MIMO-OMA," IEEE Access, vol. 4, pp. 2123-2129, May 2016.

[26] S. Ali, E. Hossain, and D. I. Kim, "Non-orthogonal multiple access (NOMA) for downlink multiuser MIMO systems: User clustering, beamforming, and power allocation," IEEE Access, vol. 5, pp. 565-577, Mar. 2017.

[27] M. Zeng, A. Yadav, O. A. Dobre, G. I. Tsiropoulos, and H. V. Poor, "On the sum rate of MIMO-NOMA and MIMO-OMA systems," IEEE Wireless Commun. Lett., vol. PP, no. 99, pp. 1-1, Jun. 2017.

[28] J. Choi, "Power allocation for max-sum rate and max-min rate proportional fairness in NOMA," IEEE Commun. Lett., vol. 20, no. 10, pp. 2055-2058, Oct. 2016

[29] Z. Ding and H. V. Poor, "Design of massive-MIMO-NOMA with limited feedback," IEEE Signal Processing Lett., vol. 23, no. 5, pp. 629-633, May 2016.

[30] X. Liu and X. Wang, "Efficient antenna selection and user scheduling in 5G massive MIMO-NOMA system,' in IEEE VTC 16' Spring, May 2016, pp. 1-5.

[31] X. Ge, S. Tu, G. Mao, C. X. Wang, and T. Han, "5G ultra-dense cellular networks," IEEE Wireless Commun., vol. 23, no. 1, pp. 72-79, Feb. 2016.

[32] R. Rolny, J. Wagner, C. Esli, and A. Wittneben, "Distributed gain matrix optimization in non-regenerative MIMO relay networks," in 2009 Conference Record of the Forty-Third Asilomar Conference on Signals, Systems and Computers, Nov. 2009, pp. 1503-1507.

[33] W. Guan and H. Luo, "Joint MMSE transceiver design in nonregenerative MIMO relay systems," IEEE Commun. Lett., vol. 12, no. 7, pp. 517-519, Jul. 2008

[34] H. Stefanović, V. Stanković, M. Č. Stefanović, P. Spalević, S. R Panić, and S. Milosavljević, "Multi-user MIMO MMSE non-regenerative relaying using local channel state information," EURASIP J. Adv. Signal Process., vol. 2012, no. 1, pp. 1-8, 2012.

[35] A. Kuhestani, A. Mohammadi, and M. Noori, "Optimal power allocation to improve secrecy performance of non-regenerative cooperative systems using an untrusted relay," IET Commun., vol. 10, no. 8, pp. 962-968, May 2016.

[36] M. A. M. Sadr, B. Mahboobi, S. Mehrizi, M. A. Attari, and M. Ardebilipour, "Stochastic robust collaborative beamforming: Nonregenerative relay," IEEE Trans. Commun., vol. 64, no. 3, pp. 947-958, Mar. 2016.

[37] S. Zhang, B. Di, L. Song, and Y. Li, "Sub-channel and power allocation for non-orthogonal multiple access relay networks with amplify-andforward protocol," IEEE Trans. Wireless Commun., vol. 16, no. 4, pp. 2249-2261, Apr. 2017

[38] S. Luo and K. C. Teh, "Adaptive transmission for cooperative NOMA system with buffer-aided relaying," IEEE Commun. Lett., vol. 21, no. 4, pp. 937-940, Apr. 2017. 
[39] E. Björnson, E. G. Larsson, and T. L. Marzetta, "Massive MIMO: Ten myths and one critical question," IEEE Commun. Mag., vol. 54, no. 2, pp. 114-123, Feb. 2016.

[40] E. G. Larsson, O. Edfors, F. Tufvesson, and T. L. Marzetta, "Massive MIMO for next generation wireless systems," IEEE Commun. Mag., vol. 52, no. 2, pp. 186-195, Feb. 2014.

[41] T. S. Rappaport, S. Sun, R. Mayzus, H. Zhao, Y. Azar, K. Wang, G. N. Wong, J. K. Schulz, M. Samimi, and F. Gutierrez, "Millimeter wave mobile communications for $5 \mathrm{G}$ cellular: It will work!" IEEE Access, vol. 1, pp. 335-349, May 2013.

[42] D. Zhang, Z. Zhou, C. Xu, Y. Zhang, J. Rodriguez, and T. Sato, "Capacity analysis of non-orthogonal multiple access with mmwave Massive MIMO systems," IEEE J. Sel. Areas Commun., vol. 35, no. 7, pp. 1606-1618, Jul. 2017.

[43] W. S. Soh and H. S. Kim, "Dynamic guard bandwidth scheme for wireless broadband networks," in IEEE Infocom, vol. 1, Apr. 2001, pp. $572-581$

[44] S. Ai, L. Jiao, F. Y. Li, and M. Radin, "Channel aggregation with guardband in D-OFDM based CRNs: Modeling and performance evaluation," in IEEE WCNC, Apr. 2016, pp. 1-6.

[45] H. A. B. Salameh, M. Krunz, and D. Manzi, "Spectrum bonding and aggregation with guard-band awareness in cognitive radio networks," IEEE Trans. Mobile Comput., vol. 13, no. 3, pp. 569-581, Mar. 2014.

[46] K. R. Budhathoki, M. Maleki, and H. R. Bahrami, "Iterative source and relay precoder design for non-regenerative MIMO cognitive relay systems," IEEE Trans. Commun., vol. 63, no. 10, pp. 3497-3510, Oct. 2015.

[47] S. Jin, M. R. McKay, K. K. Wong, and X. Gao, "Transmit beamforming in rayleigh product MIMO channels: Capacity and performance analysis," IEEE Trans. Signal Process, vol. 56, no. 10, pp. 5204-5221, Oct. 2008.

[48] J. Zhang, C. K. Wen, S. Jin, X. Gao, and K. K. Wong, "On capacity of large-scale mimo multiple access channels with distributed sets of correlated antennas," IEEE J. Sel. Areas Commun., vol. 31, no. 2, pp. 133-148, Feb. 2013.

[49] R. Couillet, J. Silverstein, Z. Bai, and M. Debbah, "Eigen-inference for energy estimation of multiple sources," IEEE Trans. Inf. Theory, vol. 57, no. 4, pp. 2420-2439, April 2011.

[50] X. Guo, Z. Niu, S. Zhou, and P. R. Kumar, "Delay-constrained energyoptimal base station sleeping control," IEEE J. Sel. Areas Commun., vol. 34, no. 5, pp. 1073-1085, May 2016.

[51] J. Proakis and M. Salehi, Digital Communications, 5th Edition. NY, USA: McGraw-hill, 2008.

[52] M. Banna and F. Merlevede, "Limiting spectral distribution of large sample covariance matrices associated with a class of stationary processes," J. Theor. Probab., vol. 28, no. 2, pp. 745-783, 2015.

[53] T. Marzetta, "Noncooperative cellular wireless with unlimited numbers of base station antennas," IEEE Trans. Wireless Commun., vol. 9, no. 11, pp. 3590-3600, Nov. 2010.

[54] A. H. Mehana and A. Nosratinia, "Diversity of MMSE MIMO receivers," IEEE Trans. Inf. Theory, vol. 58, no. 11, pp. 6788-6805, Nov. 2012.

[55] R. Couillet and M. Debbah, Random Matrix Methods for Wireless Communications. Cambridge, UK: Cambridge University Press, 2011.

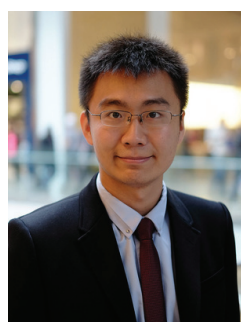

Yuanwei Liu (S'13-M'16) is a lecturer (Assistant Professor) in the School of Electronic Engineering and Computer Science at Queen Mary University of London since September 2017. He was with the Department of Informatics at King's College London from September 2016 to August 2017, where he was a Post-Doctoral Research Fellow. He received his Ph.D. degree in electrical engineering from the Queen Mary University of London, U.K., in 2016. Before that, he received his M.S. degree and B.S. degree from the Beijing University of Posts and Telecommunications in 2014 and 2011, respectively.

His research interests include $5 \mathrm{G}$ wireless networks, Internet of Things (IoT), stochastic geometry, and matching theory. He received the Exemplary Reviewer Certificate of the IEEE Wireless Communication Letter in 2015 and the IEEE Transactions on Communications in 2017. He has served as a TPC Member for many IEEE conferences, such as GLOBECOM and VTC. He currently serves as an Editor of IEEE Communications Letters and IEEE Access.

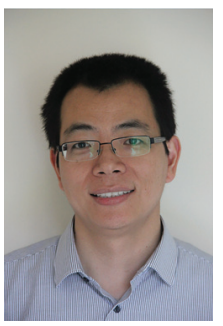

Zhiguo Ding (S'03-M'05) received his B.Eng in Electrical Engineering from the Beijing University of Posts and Telecommunications in 2000, and the $\mathrm{Ph} . \mathrm{D}$ degree in Electrical Engineering from Imperial College London in 2005. From Jul. 2005 to Aug. 2014, he was working in Queen's University Belfast, Imperial College and Newcastle University. Since Sept. 2014, he has been with Lancaster University as a Chair Professor. From Oct. 2012 to Sept. 2016, he has also been an academic visitor in Princeton University.

Dr Ding' research interests are 5G networks, game theory, cooperative and energy harvesting networks and statistical signal processing. He is serving as an Editor for IEEE Transactions on Communications, IEEE Transactions on Vehicular Technology, and Journal of Wireless Communications and Mobile Computing, and was an Editor for IEEE Wireless Communication Letters, IEEE Communication Letters from 2013 to 2016. He received the best paper award in IET Comm. Conf. on Wireless, Mobile and Computing, 2009, IEEE Communication Letter Exemplary Reviewer 2012, and the EU Marie Curie Fellowship 2012-2014.

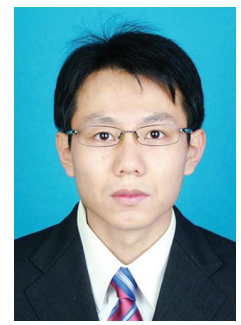

Zhenyu Zhou (S'06-M'11) received the M.E. and Ph.D. degrees from Waseda University, Tokyo, Japan, in 2008 and 2011, respectively. From April 2012 to March 2013, he was the Chief Researcher with the Department of Technology, KDDI. Since 2013, he has been an Associate Professor with the School of Electrical and Electronic Engineering, North China Electric Power University, Beijing, China. He has also been a Visiting Scholar with Tsinghua-Hitachi Joint Lab on EnvironmentHarmonious ICT, Tsinghua University, since 2014 His research interests include green communications and smart grids. Dr. Zhou served as an Associate Editor for IEEE Access, a Guest Editor for IEEE Communications Magazine, workshop co-Chair for the 2015 IEEE International Symposium on Autonomous Decentralized Systems, and a TPC member for the 2017 IEEE Vehicular Technology Conference (VTC), IEEE VTC 2016, the 2016 IEEE International Conference on Communications (ICC), IEEE ICC 2015, Globecom 2015, etc. He received the "Young Researcher Encouragement Award" from the IEEE Vehicular Technology Society in 2009 and "Beijing Outstanding Young Talent" from Beijing Government, China. processing.
Di Zhang (S'13) is a Ph. D. candidate in Waseda the Dity, Tokyo, Japan (2013-2017). He received Alternate Electrical Power System with Renewable Engineering, North China Electric Power Univer interests include 


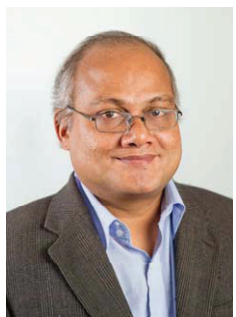

Arumugam Nallanathan (S'97-M'00-SM'05F'17) is Professor of Wireless Communications in the School of Electronic Engineering and Computer Science at Queen Mary University of London since September 2017. He was with the Department of Informatics at King's College London from December 2007 to August 2017, where he was Professor of Wireless Communications from April 2013 to August 2017. He was an Assistant Professor in the Department of Electrical and Computer Engineering, National University of Singapore from August 2000 to December 2007. His research interests include 5G Wireless Networks, Internet of Things (IoT) and Molecular Communications. He published more than 350 technical papers in scientific journals and international conferences.

$\mathrm{He}$ is a co-recipient of the Best Paper Award presented at the IEEE International Conference on Communications 2016 (ICC2016) and IEEE International Conference on Ultra-Wideband 2007 (ICUWB 2007). He is an IEEE Distinguished Lecturer. He has been selected as a Web of Science (ISI) Highly Cited Researcher in 2016. He is an Editor for IEEE Transactions on Communications and IEEE Transactions on Vehicular Technology. He was an Editor for IEEE Transactions on Wireless Communications (2006-2011), IEEE Wireless Communications Letters and IEEE Signal Processing Letters. $\mathrm{He}$ served as the Chair for the Signal Processing and Communication Electronics Technical Committee of IEEE Communications Society and Technical Program Chair and member of Technical Program Committees in numerous IEEE conferences. He received the IEEE Communications Society SPCE outstanding service award 2012 and IEEE Communications Society RCC outstanding service award 2014.

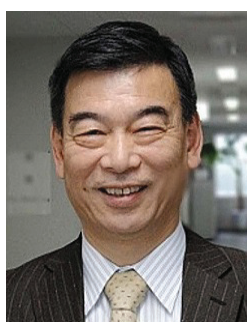

Takuro Sato (F'13) (t-sato@ waseda.jp) received his $\mathrm{PhD}$. degrees in electronics engineering from Niigata University, Niigata, Japan, in 1993 . He joined the Niigata Institute of Technology from 1995 as a Professor where his research focused on CDMA, OFDM, personal communication systems and other related areas. In 2004, he joined GITS/GITI, Waseda University, Tokyo, as a professor and currently serving as the Dean. He has engaged in the research on PCM transmission equipment development, mobile communications, data transmission and digital signal processing. He has developed the wideband CDMA system for personal communication system and joined the PCS standardization committee both in the USA and Japan. His contributes are mainly in high speed cellular modem standardization for ITU, $2.4 \mathrm{GHz}$ PCS for ITA and wireless LAN of IEEE 802.11. He has authored 11 Books and more than 200 papers. His current research interests include the next generation mobile communications, wireless communications, ICN/CCN technology, ICT in Smart Grid and their global standardizations. He served as Chair of IEEE ISADS 2015, IEEE Globecom 2015 and other various conference Chairs and journal editors. He is a Fellow of IEEE and IEICE. 